This item was submitted to Loughborough's Research Repository by the author.

Items in Figshare are protected by copyright, with all rights reserved, unless otherwise indicated.

\title{
3D reconstruction and measurement of surface defects in prefabricated elements using point clouds
}

PLEASE CITE THE PUBLISHED VERSION

https://doi.org/10.1061/(ASCE)CP.1943-5487.0000920

\section{PUBLISHER}

American Society of Civil Engineers

\section{VERSION}

AM (Accepted Manuscript)

\section{PUBLISHER STATEMENT}

This material may be downloaded for personal use only. Any other use requires prior permission of the American Society of Civil Engineers. This material may be found at https://ascelibrary.org/doi/abs/10.1061/(ASCE)CP.1943-5487.0000920.

\section{LICENCE}

In Copyright

\section{REPOSITORY RECORD}

Xu, Zhao, Rui Kang, and Ruodan Lu. 2020. "3D Reconstruction and Measurement of Surface Defects in Prefabricated Elements Using Point Clouds". Loughborough University. https://hdl.handle.net/2134/12278081.v1. 
$13 D$ reconstruction and measurement of surface defects in prefabricated elements

\section{using point clouds}

\section{Zhao Xu1 ${ }^{*}$, Rui Kang ${ }^{2}$, Ruodan $\mathrm{Lu}^{3}$}

the market. Laser scanning has already been adopted in civil engineering to reconstruct 3D model of structure and monitoring the deformation and so on. This paper seeks to explore a more automated and accurate quality control process focusing on the surface defects in prefabricated elements. Laser scanning is adopted for data collection and the 3D reconstruction of the prefabricated components. Besides, a new point cloud pre-processing, involving the KNN algorithm, reduction of data dimension and data gridding, is developed to improve the efficiency and accuracy of subsequent algorithms. The Delaunay triangle is used to extract the contour of the point cloud, then the contour is fitted to further determine the geometric data. Meanwhile, a comprehensive quality control system of prefabricated components based on relevant specifications is proposed, and the quality of prefabricated components is monitored intuitively by the values of indicators. In order to integrate into the BIM platform and better store the obtained quality information, the production quality information is designed to be extended to the IFC standard. The proposed approach will be applied to analyze the causes of quality problems in the production process and strengthen the quality control. This study designs a more efficient and accurate quality evaluation process, including data collection, data processing, indicator calculation and quality evaluation. Moreover, the results forward can feedback to the cause of the quality issues, and further improve the production quality of prefabricated elements.

Keywords: prefabrication, point cloud, surface defect, 3D reconstruction, measurement

1 Associate Professor at the Civil Engineering Dept, Southeast University, Nanjing, China. E-mail: bernardos@163.com

${ }^{2}$ Student at the Civil Engineering Dept, Southeast University, Nanjing, China. E-mail: kr97102@163.com

${ }^{3}$ Assistant Professor at School of Architecture, Building and Civil Engineering, Loughborough University, Loughborough, United Kingdom. E-mail: r.lu@lboro.ac.uk 


\section{Introduction}

Due to higher efficiency and lower cost, prefabricated construction is gradually gaining acceptance within the market (Shahzad et al. 2014; Jaillon et al. 2009). The market share of prefabricated buildings is increasing rapidly Quality issues caused by precast elements always lead to delay and additional cost for fixing. The dimensional error and surface defect are the two significant quality issues of prefabricated components, which lead to costly and timeconsuming rework. Thus, these two prominent quality issues should be addressed at an early stage.

The relevant standards, such as the guidelines for the construction, the quality control manual for the plant and the production of precast and prestressed concrete elements provide a basis for the quality judgment in the production and construction of prefabricated buildings. At the same time, during the construction of prefabricated buildings, the application of BIM strengthened the precise control of on-site construction. (Graham et al. 2018). Although some related studies have paid attention to the quality control methods of prefabricated buildings, the quality evaluation of size errors and surface defects are mostly implemented manually by measuring tapes, straightedges and so on, which leads to inaccurate and inefficient quality evaluation (Latimer et al. 2002). Meanwhile, manually measured data cannot be automatically linked to the BIM platform, which will cause additional data conversion steps and take up another data storage space. Hence, it is urgent to propose an accurate and automatic method to meet the quality control needs of prefabricated components and determine a unified data format. This article uses the IFC standard which is common to the BIM platform.

Advanced 3D reconstruction technology can collect detailed data such as geometric information of prefabricated components. Combining these data with relevant standards for interpretation can make more effective use of these data for analysis. Through these analyses, the quality management level of prefabricated components can be improved, thereby promoting the development of the industry. In the existing research, many studies have demonstrated the feasibility of 3D reconstruction for quality management applications. Laser scanning has already been adopted in civil engineering to reconstruct 3D model of structure (Bernardini and Rushmeier, 2002) and 
monitoring the deformation (Park et al. 2007) and so on. However, laser scanning is less used in prefabricated buildings, and the algorithm applied for the combination of point clouds and prefabricated components is complicate to achieve (Kim et al. 2014). Thus, a user-friendly method is needed to promote the widespread use of 3D reconstruction.

In order to overcome the above-mentioned limitations, this research proposes a quality information extraction method for the point cloud of prefabricated components. At the same time, this method refers to relevant specifications, and finally integrates into the BIM platform through the IFC data standard. First, this paper focuses on the relative standards to make sure what kinds of data quality control needs. Then, the core processing algorithm is applied to deal with point cloud of prefabricated components. Finally, the study conducts compliance verification to evaluate the processed data and stores it in BIM compatible way. This paper proposes an accurate and efficient automation to achieve prefabricated components quality control.

\section{Literature review}

\section{Laser scanning and Building Information Model}

3D reconstruction technologies can perform Non-Destructive Tests (NDT) (Rodríguez-Gonzálvez et al. 2017) and measure necessary data for quality control. As previously mentioned, there are many methods for surface noncontact detection such as image identify (Cha et al. 2017), infrared detection (Cheng et al. 2008), ultrasonic flaw detection (Sambath et al. 2010), and point cloud detection. Some studies have attempted to apply methods of surface non-contact detection to the construction industry. For example, infrared scanning can be used to find important defects such as cracks on the walls or other parts of buildings (Edis et al. 2015). It is widely applied in defect detection, but it does not do well enough in terms of measurement. Besides, photogrammetry can use images to rebuild terrain or buildings (Eulitz and Reiss, 2015). It is suitable for large scale data collecting, but it can hardly satisfy the high accuracy requirement when altered for the micro level. Moreover, it is easily affected when the environment is not ideal. Because of these limitations, these methods have not yet been widely applied to 
prefabricated components. However, laser scanning is a more stable and adaptable measurement compared with infrared scanning and photogrammetry. Recording data by laser scanning makes the data processing more convenient. Furthermore, an advanced laser scanner can provide high accuracy.

Laser scanning is not the only technology that impacts on the development of Architecture, Engineering \& Construction (AEC). With the increase of data and management requirement, building information model (BIM) arises to storage and exchange information. BIM has made the information exchange and management smoother in the AEC field. Many scholars in the industry are working on semantic enrichment to describe more complete information (Belsky et al. 2016). And BIM is playing an increasingly important role in the life cycle of building owing to its expandability. Some technologies have been developed around BIM such as RFID (Demiralp et al. 2012) which makes the information traceable. Among the many data exchange formats used in the BIM platform, IFC (Industry Foundation Classes) is the most widely used and the most compatible. IFC is the standard for product data exchange and sharing. The language it uses is EXPRESS, which is an object-oriented, standardized data description language that focuses on the description and definition of data and its relationships. Venugopal et al. (2012) and Aram et al. (2013) have already proved the interoperability of IFC but focus less on quality control information. Applying laser scanning and enrich the IFC format can further improve the performance of quality control.

\section{$3 D$ reconstruction and quality control using point cloud}

Point cloud is actually a set of data points exist along with the coordinates within the 3D scanned space. The precision of professional equipment, which can reach $0.01 \mathrm{~mm}$ level, can meet most industrial needs (Boehler et al. 2003). At the same time, research on the scanning accuracy (Kovacs et al. 2006) and the development of the high precision scanner system (Blais et al. 1988) has also been followed up to ensure accuracy of point cloud. Based on these studies, surface reconstruction using laser scanning can be well performed to achieve multiple purpose (Dey, 
reconstruction was used to extract information becomes popular to fully use the accuracy of point cloud (Pu and

Vosselman, 2009). When using point cloud data to reconstruct a separate structure, some algorithms can be used to extract important information from the reconstructed model for subsequent analysis. This information can be combined with BIM in AEC field for quality inspection to further improve the management of prefabricated buildings.

Due to the advantages of point cloud data in efficiency, safety and economy, laser scanning has been widely used to measure and collect data in the on-site construction stage of prefabricated buildings and the maintenance stage of buildings and bridges. For example, Cong (2018) has used laser scanning for on-site dimensional inspection of industrial plant piping systems. It is significant to extract pipeline from point cloud data and then achieve inspection. Sacks et al. (2018) use laser scanning to obtain the point cloud data of bridge in the operation and maintenance period and further apply digital twin to form models for the purpose of inspection. These studies have proven the feasibility of using laser scanning to obtain point cloud data for quality monitoring.

There are also studies applying laser scanning on the quality control of prefabricated components. Kim et al. (2016) use a coordinate transformation algorithm to achieve non-contact dimensional quality assurance and then analysis the point cloud data to gain quality information; Nahangi et al. (2015) use images to obtain point cloud data and conduct 3D registration with original CAD drawing for automated discrepancy qualification; Wang et al. (2019) uses the scan-to-BIM framework to create an as-is BIM model of a precast panel for geometry quality assessment. These studies applied the existing technology to the quality monitoring of prefabricated components and proposed solutions for automated inspection but did not further analyze them in conjunction with relevant specifications. In this paper, a new point cloud processing method is proposed with reference to the specification, and different algorithms are used in combination with the characteristics of prefabricated components to improve the feasibility and application efficiency of the algorithm. 
After the scene is scanned and the point cloud data is obtained, before the data format is converted into the BIM technology common format, the source data can be processed to gain the geometric information. After extracting the contour using the existing technology, the data related to the quality control of the prefabricated component can be further calculated. (2016) scored a contour by a binary classifier, and then looks for points similar to the highly probable point to further determine the contour shape. In this way, the contours in the 3D point cloud of the building can be effectively extracted; Javidrad et al. (2011) sliced the 3D point cloud and filtered the 2D points. Then, the B-spline curve was curves. Boulaassal et al. (2009) and Awrangjeb (2016) used the Delaunay triangle for contour extraction and both achieved good results. defect detection; Schnabel et al. (2007) proposed a method to extract building geometries automatically from unorganized point cloud, which reduced manual work to create as-is BIM. Dimitrov \& Golparvar-Fard (2015) solved the problem of complex point cloud segmentation in a single scene, provide a solution for building point building after the data de-noising and region growing; Zhou et al. (2015) and Kalasapudi et al. (2014) respectively propose the fitting method after boundary extraction, and the alpha shape algorithm used by Zhou is also applied in this paper in another way. All the researches mentioned above have provided theoretical implementation for feature extraction of building point cloud, and provide references for the process of this article. 
the efficient information management of BIM. Although point cloud contour extraction has been applied in many

141 different fields and solved some issues in the AEC field, it focused on the defect detection area or as-is building

rebuild. It remains a research gap in the combination of point cloud contour extraction and prefabricated building quality management. control in the product process was identified. Furthermore, the research on the cutting-edge technologies of 3D reconstruction has proved that it is feasible to apply laser scanning to replace manual measurement and achieve better quality control. However, it should be noted that point cloud must be combined with appropriate management methods and related requirements to gain more efficient and accurate automatic inspection. Thus, how the point cloud method is applied according to prefabricated component structure features and combined with a management system is the key to filling the gap.

\section{Methodology} accuracy of production quality control. The methodology starts with the data pre-processing. On the premise of retaining the edge features of data for subsequence calculation and reducing the interference of noises and the number of data points, the K-Nearest Neighbor $(\mathrm{KNN})$ algorithm is adopted. Then in step 1, the data is transformed from $3 \mathrm{D}$ into the 2D, by this way the difficulty of complex spatial topological relationships can be reduced. When proceeding to the step 2, two technical routes are needed. First route, the boundary of data is extracted by Delaunay triangle mesh and Alpha Shape algorithm, and then the curve is fitted by least squares method to obtain detailed geometric information in step 3 . The other route, the defect in the point cloud data is located by gridding and the Delaunay triangle and subsequent algorithms are used to extract the defect area. In the end, the result is carried out to be verified with prefabricated component standards. The overall research methodology is presented in Figure 1. 


\section{Prefabricated component quality standard}

In order to perform the quantitative analysis of the evaluation indexes for production quality, an evaluation specification should be determined first. By referring to various specifications for quality evaluation of precast concrete components (Guidelines for the use of structural precast concrete in buildings; Manual for quality control for plants and production of precast and prestressed concrete products: PCI MNL-116; Specifications for tolerances for concrete construction and materials and commentary: ACI 117M-2010; Tolerances for building part1: precast ordinary, reinforced and prestressed concrete components: DIN 18203-1997; PCI design hand book-precast prestressed concrete: PCI MNL-120-04; Tolerance manual for precast and prestressed concrete construction: $M N L$ 135-00; Specification for tolerances for concrete construction and materials and commentary: ACI 117), it can be found that the allowable deviations vary in different specifications for components of the same category and the same volume. This paper designed the evaluation indicators based on these existing specifications implemented in AEC industry.

In addition to conventional geometric information including length, width, and height, diagonal length is also considered as an important evaluation indicator. Considering the advantages and disadvantages of each specification, this research proposes the allowable deviation for production quality evaluation based on the geometric information automatically extracted from 3D reconstruction of prefabricated concrete components in Table 1 and the defect on the surface (such as honeycomb, surface voids, crack, etc.) in Table 2. Tables 1 and 2 set criteria for evaluating production accuracy and surface defects, so the physical value of quality evaluation index calculated by the algorithm can be quantitatively analyzed.

\section{Point cloud data pre-processing}

The original point cloud is composed of a large number of scattered points, including the noise generated in the acquisition process, which makes it difficult to use an algorithm to process the original point cloud data. Therefore, the data needs to be preprocessed through three steps, namely, the KNN algorithm, reduction of data 
dimension and data gridding. The KNN algorithm can help reduce noise to eliminate interference, which can

187 facilitate the application of the following algorithm. Next, the original data dimension needs to be reduced,

providing the basis for the subsequent studies. After reducing the dimensions of the data to $2 \mathrm{D}$, inspired by Wack and Wimmer (2002), the gridding of point cloud data then be applied, which is also an important step of data preprocessing. Gridding can pinpoint defect and reduce the amount of point cloud data that needs to be calculated. laser scanning as a preprocess method. The core of $\mathrm{KNN}$ classification algorithm is to predict the classification of a new data point based on a dataset in which the data points are divided into several categories. In other words, a data point is classified by a plurality vote of its neighbors, with the data point being assigned to the class most noise. Because laser scanning focuses on features such as boundaries, the density of boundary points and the number dataset, if the distance from other points exceeds this threshold, it will be considered as noise and deleted. This is the de-noising process. The neighboring points of the noise are obviously less than the planar points, so the $\mathrm{K}$ value of neighboring points are much larger than those of planar points and noise. At the same time, through different values of K, KNN algorithm calculates a threshold to judge a data point as an outlier (noise). For a data point in the used to remove the noise is smaller than that of down sampling the planar points. Under this premise, when the appropriate $\mathrm{K}$ value is selected for the down-sampling process which is the process to reduce the number of planar points, the de-noising process is also completed at the same time. It can be seen from the principle of KNN algorithm that the value of $\mathrm{K}$ has a great influence on the results. Therefore, when applying the $\mathrm{KNN}$ algorithm, the value of K should be considered carefully and determined by comparison.

Point cloud data after data cleaning is still three-dimensional data, so the second step for reduction of data dimension is applied. After fully verifying the possibility of data dimension reduction, this research conducted 
dimension reduction for the three-dimensional data. Due to the geometric properties of prefabricated components,

the collected point cloud forming the surface of the component can be surrounded by a cuboid with tiny height.

Therefore, it is in line with the practical application of this research that these data points are extracted after three points determine a certain plane to form a surrounding cuboid so that the three-dimensional can be reduced to twodimensional. After following verification, the error caused by dimensionality reduction does not affect the defect detection results as shown in Figure 2. are randomly selected multiple times by the computer according to the principle of non-collinearity. Finally, among the results of multiple selections, the one with the largest number of points is taken. This is because the point clouds

Subsequently, a rectangular bounding box with the plane represented by the equation as the central plane is established. The height of the bounding box is a tiny value that is adjustable to control the number of data points bounded. The data points in the bounding box are considered to form a plane and separately extracted for subsequent processing as independent plane files. Then the extracted points are rotated so that the plane they form is parallel to the $\mathrm{x}-\mathrm{y}$ plane to eliminate the $\mathrm{z}$ coordinates. One of the rotation matrices that rotates around axis is as follows:

$$
R_{x}\left(\theta_{x}\right)=\left[\begin{array}{ccc}
1 & 0 & 0 \\
0 & \cos \theta_{x} & \sin \theta_{x} \\
0 & -\sin \theta_{x} & \cos \theta_{x}
\end{array}\right]
$$

After rotating the original three-dimensional data points around the $\mathrm{x}$ and $\mathrm{y}$ axis by $\theta_{x}$ and $\theta_{y}$, the $(\mathrm{x}, \mathrm{y})$ coordinates in the three-dimensional data are extracted separately to form $2 \mathrm{D}$ data, so as to reduce the data dimensionality. In rotation, there is an error angle $\varphi$, making the approximate plane of the planar point after rotation 
cannot be completely parallel to the $x-y$ plane, as shown in Figure 3.

With respect to the existence of error angle $\varphi$, extracting $(\mathrm{x}, \mathrm{y})$ coordinates in the process of dimension

reduction to eliminate $\mathrm{z}$ coordinates is equivalent to mapping points on the original plane to the $\mathrm{x}-\mathrm{y}$ plane, resulting in an error between the length $L^{\prime}$ and the actual length $L$, which can be calculated as follows: components is $400 \mathrm{~mm}$, if the error angle is controlled at $1^{\circ}$, which can be limited to, the resulting error $\Delta \mathrm{L}$ is gridding to pinpoint the location of defect. deviation $1 \mathrm{~mm}$. Due to transportation and production conditions, most of the prefabricated components that are conventionally used are less than 6 meters in length. When the size is enlarged to 6 meters, the error is still less than $1 \mathrm{~mm}$. Therefore, the impact of errors in data dimension reduction can be neglected.

In the second step, data dimension reduction was completed by using the geometric advantages of prefabricated components and transformed the three-dimensional problem into a two-dimensional data processing problem. After data dimension reduction, surface defect problem is further simplified.

In the third step, data gridding is used to pinpoint the location of defect and further extract the defect area for separate calculation as shown in Figure 4. For the planar extraction data of the entire prefabricated component, the surface defect typically only occurs in a small area. In this case, it is not necessary to calculate the whole area. Another method that can be used to locate defect is relative coordinate system of the prefabricated component. However, setting the coordinate system requires additional work, and the defect area is often irregular. Using the coordinate system to extract defect requires relatively complicated calculations. Therefore, this paper adopts data

According to the ( $\mathrm{x}, \mathrm{y})$ coordinates of the point cloud data, proper $\mathrm{M}$ and $\mathrm{N}$ are select to form a $\mathrm{M} \times \mathrm{N}$ grid. Considering the area and location of the defect, the grid partition can be adjusted to precisely locate the defect. At 
the same time, the divided grid is output in blocks, and the defected grid is separately extracted to further calculate.

\section{Data boundary extraction and fitting}

This section is focusing on the dimensional error. Figure 5 shows the processing flow of boundary information.

First, Delaunay triangulation algorithm was used to form disjoint triangular grids for the scattered points of point cloud data after dimension reduction. The three vertices of each triangle are extracted for subsequent calculation for boundary judgment. To determine the boundary, the parameter $r$ is calculated by Alpha Shape algorithm. The boundary can be judged by being compared with the parameter developed. Finally, the obtained boundary points are fitted to obtain the dimension and quality information of precast concrete components. triangulation algorithm is adopted in this paper to form triangular grids. is used to calculate the planar triangular grids of prefabricated components in this research. The Lawson algorithm first creates a large triangle to surround all the data points; followed by inserting points into the triangle and connecting them to the vertices of the triangle to form a new triangle; and finally performs the empty circumcircle test one by one, generating initial triangular grids. Meanwhile, the triangulation network is verified as Delaunay triangulation network by switching diagonals. 
Figure 6(a) shows the principle of Delaunay algorithm. $\triangle \mathrm{ABC}$ is the largest triangle surrounding the data point

at the beginning, $\mathrm{P} 1, \mathrm{P} 2$, and $\mathrm{P} 3$ are the points inside the triangle. $\mathrm{P} 2$ is in the circumcircle of $\triangle \mathrm{ACP}_{1}$, so the triangle is not a Delaunay triangle and is removed from the grid. Figure 6 (b) is a Delaunay triangulation grid formed by processing the two-dimensional plane extracted from the prefabricated concrete staircase by the algorithm. As can be seen from the results, the generated triangular grid satisfies the Delaunay triangulation criteria. However, such triangular grid cannot directly extract the dimension information, making boundary judgment and extraction required based on the Delaunay triangular grid.

(2) Boundary judgment based on Alpha Shape algorithm

Alpha Shape algorithm were first defined by Edelsbrunner et al. (1983), which is an improved algorithm for the convex hull boundary algorithm. In essence, it selects a circle with a fixed radius and judges every pair of possible boundary line on the circle in the data points. If there are no other points in the circle except these two points, it is judged as the boundary. According to different values of the key parameter $\alpha$, the boundary data points that can be judged by this algorithm are different. Thus, the value of $\alpha$ need to be selected appropriately by comparison. According to the principle of the Alpha Shape algorithm, it is necessary to iteratively check all the possibilities of forming a boundary, which takes a significant amount of time. Therefore, Delaunay triangulation is often performed before applying the algorithm, so as to preprocess the data more effectively.

The application of the Alpha Shape algorithm is closely related to the set of triangular points in Delaunay triangular grid. It can be known that each triangle in the grid has its own characteristic radius, which is the radius of the minimum circumscribed empty circle of the triangle. According to the principle of the Alpha Shape algorithm, the question whether there are other points in the circle with fixed radius can be converted into the question whether the minimum circumradius of the Delaunay triangle is larger than the set radius. Therefore, to determine whether the triangle is a boundary triangle, it only needs to determine the size relation between the radius of the minimum circumscribed empty circle and the set radius. Thus, the geometric information of each Delaunay triangle needs to 
first be obtained. The formulas of the side length and the semi-perimeter of triangle are shown as follows:

$$
\begin{gathered}
l=\sqrt{\left(x_{1}-x_{2}\right)^{2}+\left(y_{1}-y_{2}\right)^{2}} \\
s=\frac{1}{2}(a+b+c)
\end{gathered}
$$

where $\left(\mathrm{x}_{1}, \mathrm{y}_{1}\right)$ and $\left(\mathrm{x}_{2}, \mathrm{y}_{2}\right)$ are the endpoints coordinates of the calculated side; and $\mathrm{a}, \mathrm{b}$, and $\mathrm{c}$ are the lengths of the

three sides of the triangle. Using this geometric information, the area of the triangle can be calculated with the help

of the Heron formula, and the radius of the minimum circumscribed empty circle of the triangle can then be obtained,

as shown in the following formulas:

$$
\begin{gathered}
S=\sqrt{s(s-a)(s-b)(s-c)} \\
r=\frac{a \times b \times c}{4 \times S}
\end{gathered}
$$

Finally, based on the relationship between $\mathrm{r}$ and $1 / \alpha$, it can be determined whether the triangle is a boundary triangle:

The result of processing the obtained data by using the Alpha Shape algorithm is shown in Figure 7.

(3) Data boundary fitting based on least squares method

After the boundary points of precast concrete are obtained, the least square method is used for boundary fitting. The least square method seeks the best function matching of the data by minimizing the sum of squared errors. Due to the geometry of the prefabricated elements, the lines are basically straight except for the reserved holes. Except for the hole boundary, the boundary points of the four edges are fitted to the straight-line equations after truncating the end points. The application process of straight-line method is relatively simple, so the boundary fitting of this part is not listed. The process of applying the least square method to a closed curve is complicated, which needs to conduct derivative and find the pole. Therefore, this section will focus on the process and formula for fitting circular 
value is a non-negative number. The formula is as follows:

$$
f=\sum\left((x-A)^{2}+(y-B)^{2}-R^{2}\right)^{2}
$$

Here $(A, B)$ is the center of fitted circle while $\mathrm{R}$ is the radius of fitted circle. The actual operation to achieve data boundary fitting is to calculate (A, B) and R, and minimize f. At the same time, the equation will be too complicated

if the formula (12) is expanded, so a new squared difference formula is obtained by parameter substitution:

$$
Q(a, b, c)=\sum\left(x^{2}+y^{2}+a x+b y+c\right)^{2}
$$

The point with partial derivative of 0 is extreme point by figuring out the partial derivative of $Q(a, b, c)$. Since the squares calculation. The function values of all obtained extreme points are compared, the minimum is the solution.

$$
\left\{\begin{array}{l}
\frac{\partial Q(a, b, c)}{\partial a}=\sum 2\left(x^{2}+y^{2}+a x+b y+c\right) x=0 \\
\frac{\partial Q(a, b, c)}{\partial b}=\sum 2\left(x^{2}+y^{2}+a x+b y+c\right) y=0 \\
\frac{\partial Q(a, b, c)}{\partial c}=\sum 2\left(x^{2}+y^{2}+a x+b y+c\right)=0
\end{array}\right.
$$

The solution can be found by converting the formula to a matrix:

$$
\left[\begin{array}{l}
a \\
b \\
c
\end{array}\right]=\left[\begin{array}{ccc}
\sum x^{2} & \sum x y & \sum x \\
\sum x y & \sum y^{2} & \sum y \\
\sum x & \sum y & N
\end{array}\right]^{-1}\left[\begin{array}{c}
-\sum\left(x^{3}+x y^{2}\right) \\
-\sum\left(x^{2} y+\sum y^{3}\right) \\
-\sum\left(x^{2}+y^{2}\right)
\end{array}\right]
$$

Center and radius of the circle can be calculated based on obtained a, b, c: $A=\frac{a}{-2}, B=\frac{b}{-2}, R=\frac{1}{2} \sqrt{a^{2}+b^{2}-4 c}$. The geometric information of fitted circle is solved, and then the boundary linear equation of precast concrete is combined to check positioning information and opening size of the 
(4) Surface defect area calculation

Defect area cannot be calculated merely by data gridding. Because the defect has no fixed shape, it is still surrounded by normal data points. Therefore, it is necessary to further separate the defect data from the normal data. the geometry feature. The Vertex Clusters algorithm starts from the seed point and diverge outward through a circle of fixed radius. The points contained within the circle are judged to be the same cluster, and these points continue to spread as seed points until the circle no longer contains new points or artificial stops. Due to the application of the KNN algorithm for data cleaning, the density of the defect points exceeds the normal point, which is more the output after separating. Nevertheless, the purpose of applying these two algorithms is different from the above. The defect point does not need to be strictly extracted. The defect area is the critical point for the surface defect to match this radius, compare the results, and choose the optimal $\alpha$ value.

\section{Experimental results and analysis}

\section{Data selection}

This paper focuses on the practicality of the algorithm, so the data selection combines the actual situation and related policies and strives to represent a wide range of examples with special cases. 
The selection of data acquisition objects for concrete prefabricated components should be characterized by strong representativeness, wide application range, and good applicability. Prefabricated interior and exterior wall panels, prefabricated stair slabs, and prefabricated floor slabs (including prefabricated composite floor slabs) hereinafter referred to as "three slabs" - which are typical prefabricated components, assume important structural and functional functions. The structural importance of "three slabs" in prefabricated construction is self-evident. It can provide reference for subsequent quality monitoring of the element in practical application. Due to functional requirements of the element, there are many embedded parts on board, such as pre-embedded electric boxes and water pipes on prefabricated interior wall, combined with load-bearing structural characteristics of floor, wall, and stairs, so rebar is widely applied in the element. Due to the above characteristics, "three slabs" can make full use of prefabricated properties of the prefabricated elements in the manufacturing process. Therefore, choosing "three slabs" for data collection can ensure the collected data is representative, and the conclusions and measures can be extended to other prefabricated elements, which is universal.

(2) Data acquisition equipment

The data acquisition equipment used in tis HandySCAN BLACK. The light source are 7 blue laser crosses and the measurement rate is 800,000 measurements/s. The accuracy of this equipment can reach $0.035 \mathrm{~mm}$ and the volumetric accuracy (based on part size) is $0.020 \mathrm{~mm}+0.060 \mathrm{~mm} / \mathrm{m}$. This device can meet the needs of collecting information quickly and with high accuracy. Such accuracy can meet the needs of dimensional measurement and defect area calculation in this paper. For cracks with higher requirements, it is beyond the scope of this paper.

The main application algorithm of the laser scanner used in this data acquisition is laser triangulation method. The principle is shown in Figure 9. During the data acquisition process, the laser beam has a certain $\gamma$ angle to the surface of object, which applies oblique laser triangulation method. Reflected light is at $\alpha$ angle to surface normal, 
and at $\beta$ angle to light sensor. The distance to be measured is S, spot moving distance is S', object distance is $L$, and

image distance is L'. Combined with focal distance $\mathrm{f}$, the distance $\mathrm{S}$ to be measured:

$$
S=\frac{S^{\prime}(L-f) \sin \beta \cos \gamma}{f \sin (\alpha+\gamma) \mp S^{\prime}\left(1-\frac{f}{L}\right) \sin (\alpha+\beta+\gamma)}
$$

The coordinates of the points located on the surface of the component are two-dimensional data. Together with the distance $\mathrm{S}$ to be measured, complete three-dimensional data can be generated. The accuracy of the data acquisition can fully meet the need of quality evaluation in this way.

\section{Implementation and results} calculation and defect calculation. The reserved holes of precast concrete staircase are representative, which was used to verify the size calculation. Defect area was calculated according to the surface pockmark of prefabricated exterior walls and the angular collapse of prefabricated stairs

Using KNN algorithm to reduce number of points was the first step of data pre-processing. Since the value of

$\mathrm{K}$ has a great influence on the results, different $\mathrm{K}$ values were adopted in this research to process the point cloud data of precast concrete stairs. The results are exhibited in Table 3. According to the characteristics of the KNN algorithm, it reduced the number of points and noise under the premise of retaining features. The experimental results obtained by different $K$ values show that when $K=4$, the number of points reached the minimum. Figure 10 shows the change of the original point cloud data after down sampling. 
It can be found that $\mathrm{KNN}$ algorithm retained the geometric features of the original data, and only reduced the

411 density of excessively dense points after down sampling. The point cloud data obtained after applying KNN algorithm removed the noise, facilitating the next step of data processing. Then dimension reduction was applied to convert point cloud data to 2D, the result is shown in Figure 11. defect information can be output completely without including the normal surface in the defect information, thus providing good data for subsequent calculations of defect parameters. algorithm are significantly different from the Delaunay triangular grid formed before. Meanwhile, the precast concrete components have a large number of holes which are reserved for transportation and installation. These reserved holes are required to be accurately positioned to ensure the quality of installation, which means that the extraction of the internal boundaries is extremely important for quality detection.

However, it can be seen from Figure 13 that after using Alpha Shape algorithm, due to the excessively dense data points of the point cloud of prefabricated components and the lack of rigorous discussion for the value of $\alpha$, the extracted boundary has excessive thickness and independent interference points. Therefore, a careful comparison of different $\alpha$ values was carried out as shown in Figure 14.

It is inevitable that redundant boundary points were extracted due to the limitation of data itself. Therefore, this paper was devoted to minimize interference points to ensure that the boundary lines can be extracted as completely as possible. Relatively speaking, when $\alpha=0.85 / 0.8$, the boundary lines could be completely extracted. 
finally set as 0.85 . The final quality data can be obtained by fitting the extracted boundary points.

Then least square method was applied for boundary fitting. The four linear fitting curves on the four sides of prefabricated stair plane were obtained as follows: $X_{1}=-220.11, X_{2}=962.11, Y_{1}=201.86, Y_{2}=601.69$. $\mathrm{L}=188.22 \mathrm{~mm}$ and the width $\mathrm{H}=399.83 \mathrm{~mm}$. Data error with calibration of design $\mathrm{CAD}$ drawings was $\Delta L=1182.22-1180=2.22 \mathrm{~mm}, \Delta H=399.83-400=-0.017 \mathrm{~mm}$.

At the same time, the circle center( A, B ) of the two reserved openings obtained after fitting as $(-38.90,500.53)$, (780.24,499254). Combined with boundary positioning line, the distance between circle center and the left and right sides were $181.21 \mathrm{~mm}$ and $181.87 \mathrm{~mm}$, and the distance from top edge were $101.16 \mathrm{~mm}$ and $102.15 \mathrm{~mm}$. The radius of circles were $R_{1}=29.47 \mathrm{~mm}, R_{2}=29.45 \mathrm{~mm}, \Delta R_{1}=0.53 \mathrm{~mm}, \Delta R_{2}=0.55 \mathrm{~mm}$. Since the prefabricated stairs for scanning experiments are not perfect in size, the radius of the reserved hole is actually smaller than the design value, resulting in that the calculated data after fitting the curve is also smaller than the corresponding actual size. The original size information and the size information calculated by actual collection are marked on the map in Figure 15.

(2) Defect area calculation results

In addition to dimensional deviation in appearance quality defects, defect area is the other important evaluation index. The defect area in the surface pockmark of prefabricated exterior walls and the angular collapse of prefabricated stairs were calculated as an example. The position and size of appearance defects in the threedimensional reconstruction model both are shown in Figure 16.

By extracting the plane where defect occurs, adjusting division size of the planar grid, exporting defect grid to finally separate defect data from normal data, the defect points after separation were obtained finally. The triangular 
As shown from the comparison in Figure 18 the generated triangular grid of defect points substantially restored

the actual defect region, and scattered defect was separated from large defect, which is a prerequisite for the for the subsequent accurate calculation of the defect region area. The triangle of the damage point constitutes the entire area of the damage. Therefore, the area of each triangle was calculated separately and then summed as eq.9 above.

The results show that the selection of $\alpha=0.4$ for surface pockmark of prefabricated exterior walls to form triangular grid could meet the requirement of calculation area. The calculation results were $\mathrm{S}_{1}=56609.39 \mathrm{~mm}^{2}$ and area percentage $p_{1}=\frac{s_{1}}{S}=1.369 \%$; the selection of $\alpha=0.5$ for angular collapse of prefabricated stairs to form triangular grid, $\mathrm{S}_{2}=2266.24 \mathrm{~mm}^{2}$ and area percentage $\mathrm{P}_{2}=0.446 \%$.

\section{Verification with prefabricated component standards}

Through the establishment of index evaluation criteria and the determination of corresponding weight, the calculated results were evaluated in combination with specific criteria in Table 4. According to the weight and the evaluation value of each sub-index, namely the corresponding $0 / 1$ value, the comprehensive evaluation index value was calculated as shown in Table 5. Only when the comprehensive evaluation index is 1 , the production quality of the element is judged to be qualified. The lower comprehensive evaluation index is, the worse the production quality of the element, and the more aspects that need to be improved.

The comprehensive quality evaluation index of precast concrete stairs was 0.96 , and the quality was judged to be unqualified. The angular collapse occurred during the removal of frame exerted a great negative impact on the results of quality evaluation. Although the area ratio of angular collapse met the requirements, the number of angular collapses caused by violent construction was excessive, which led to the unqualified results of the quality evaluation.

The results of production quality comprehensive evaluation index can directly reflect whether the quality of prefabricated elements satisfy the requirements of standard, which provide a quick and intuitive information for workers to deal with in time. At the same time, the detailed measurement data of each indicator can provide support for finding the cause of the quality issues and improving production quality. 
In order to meet the compatibility of the BIM platform and better store the obtained quality information, the production quality information of the prefabricated components should be extended to the IFC standard. The extension of IFC is divided into three ways: (1) Extension based on adding new entity; (2) Extension based on

IfcProxy entity; (3) Extension based on property set. The last, which does not generate new entities nor modify the original entity with small and targeted scope of modification, was adopted in this paper. The new property sets, and property definitions were extended under the original property set definition. To perform property set definition, it is necessary to improve the property set name (PsetName), applicable entity (Entity), applicable type value reference property. the property sets definition and property sets of concrete prefabricated wall.

The EXPRESS structure diagram can intuitively express the IFC extension as shown in Figure 19. After the completion of the production quality property expansion of the concrete prefabricated components, the information collected by the previous algorithm were stored in the IFC standard, which not only enriched the semantic information of the BIM model, but also integrated the production quality information into a BIM platform as a

\section{Discussion}

The main purpose of this paper is to use high-precision scanning reconstruction technology to detect and digitize the quality problems of prefabricated components in the process of assembly and construction, to achieve higher efficiency and higher precision. In order to achieve this goal, the experiment designed in this paper uses the Delaunay triangle to extract the contour of the point cloud, and further linearly fits to determine the geometric data. 
position, shape information and appearance defect. After comparing with the design information of the prefabricated

building, the obtained difference value can more suitably reflect the quality problems of the prefabricated components in the production process. According to the error analysis and the technical precision adopted in the experiment, it can be proven that the algorithm proposed in this experiment can meet the needs of the quality management of the prefabricated building. necessary to consider the actual situation. Combined with the requirements in the quality assessment standards related to prefabricated buildings, this paper also established a comprehensive evaluation system. Using reasonable weight distribution, the data values are analyzed to obtain meaningful evaluation indicators. With these indicators, it is possible to analyze the causes of quality problems in the production process and strengthen the quality control. This will not only provide detailed data for subsequent construction, but also forward feedback to the cause of the problem, improving the quality of the prefabricated building from two aspects.

As shown in Figure 20, considering point cloud data and the design document from the structural designer as input, then extract the key information, such as geometric information, to check and calculate the needed data. The error data and defect data can then be used for quality inspection using the quality standard, for which the quality manager provided. If the prefabricated component passes the inspection, then the information is transferred to construction workers on site, otherwise it will be transferred to factory workers to further analyze the causes and 519 improve the performance.

\section{Conclusion}

At present, the quality management of prefabricated components is still limited to manual measurement, resulting in poor efficiency and accuracy. In response to such deficiencies, this paper aimed to explore a more automated and accurate quality control process through the research on 3D reconstruction technology. After 
is more adaptable. The Delaunay triangle was adopted as the core of the algorithm. The comprehensive prefabricated component quality evaluation system based on relevant specifications was established. Besides, a new process for extracting quality information was developed, which greatly improved the efficiency and accuracy.

In this process, the KNN algorithm was used to reduce the data density, successfully compressing the data to $60.20 \%$ of the original volume while retaining the data characteristics. This operation reduced the size of the data set while also increased the computational speed of subsequent algorithms. In order to realize the contour extraction of point cloud data, the Alpha Shape algorithm was applied flexibly to overcome the problem of concave plane extraction, and finally effectively separated the inner and outer boundaries. In addition, the defect information was extracted using data grid positioning integrated with the Vertex Clusters algorithm, which also provided data support for determining the values of indicators. The comprehensive data can provide detailed parameters for on-site construction, and guide the management system in the pre-production process.

This paper presented the general flow of quality management of prefabricated components, which involves plane extraction, and it was impossible to perform dimensionality reduction on the surface. Next, the shape of the point cloud acquisition, contour extraction and calculation parameters. Although the feasibility of practical application was demonstrated by typical examples, some limitations were still presented in practical application. First, the diversity was not fully considered. Because the current steps of dimensionality reduction were limited to reserved holes was also set to a regular shape, such as a perfect circle or a rectangle. However, in the case of prefabricated buildings with higher assembly rates, prefabricated components tend to diversify. Lastly, the automation operation is not fully realized, and multiple parameters need to be manually adjusted according to the actual situation, which reduced the efficiency of the process. Although a simple dimensionality reduction algorithm was adopted to improve the applicability of data calculation, a lot of work need to conducted to enhance the automation of operation in the future research. Nonetheless, this study is still valuable for improving the quality detection efficiency of prefabricated elements and filling the research gap in this area. 


\section{Data Availability Statement}

Some or all data, models, or code that support the findings of this study are available from the corresponding author upon reasonable request.

\section{Acknowledgement}

The authors' special thanks go to all survey participants and reviewers of the paper, and appreciation to the National Science Council of P. R. C. for financially supporting this research (NSFC-71302138), and fund by the Priority Academic Program Development of Jiangsu Higher Education Institutions (CE01-2-2), and support from the Scientific Research Starting Foundation for Returned Overseas Chinese Scholars, Ministry of Education China.

\section{Reference}

Altman N S. An introduction to kernel and nearest-neighbor nonparametric regression [J]. The American Statistician, 1992, 46(3): 175-185.

Aram, S., C. Eastman, R. Sacks. 2013. "Requirements for BIM platforms in the concrete reinforcement supply chain." Automation in Construction, 35, 1-17. https://doi.org/10.1016/j.autcon.2013.01.013

Awrangjeb, M. 2016. "Using point cloud data to identify, trace, and regularize the outlines of buildings". International Journal of Remote Sensing, 37(3), 551-579. https://doi.org/10.1080/01431161.2015.1131868

Belsky, M., R. Sacks, I. Brilakis. 2016. "Semantic enrichment for building information modeling”. Computer-Aided Civil and Infrastructure Engineering, 31(4), 261-274. https://doi.org/10.1111/mice.12128

Bernardini, F., H. Rushmeier. 2002. "The 3D model acquisition pipeline". Computer Graphics Forum, 21(2), 149172. https://doi.org/10.1111/1467-8659.00574

Blais, F., M. Rioux, J. A. Beraldin. 1988. "Practical considerations for a design of a high precision 3-D laser scanner system". Optomechanical and electro-optical design of industrial systems, 959, 225-246. DOI: $\underline{10.1117 / 12.947787}$

Boehler, W., M. B. Vicent, A. Marbs. 2003. "Investigating laser scanner accuracy." The International Archives of Photogrammetry Remote Sensing and Spatial Information Sciences, 34: 696-701. DOI: $\underline{10.1002 / \mathrm{pbc} . \mathrm{ABSTRACT}}$ 
Boulaassal, H., T. Landes, P. Grussenmeyer. 2009. “Automatic extraction of planar clusters and their contours on building façades recorded by terrestrial laser scanner”. International Journal of Architectural Computing, 7(1), 1-20. https://doi.org/10.1260/147807709788549411

Cha, Y. J., W. Choi, O. Büyüköztürk. 2017. "Deep learning- based crack damage detection using convolutional neural networks." Computer-Aided Civil and Infrastructure Engineering, 32(5), 361-378. https://doi.org/10.1111/mice. 12263

Cheng, C. C., T. M. Cheng. 2008. "Chiang C H. Defect detection of concrete structures using both infrared thermography and elastic waves." Automation in Construction, 18(1): 87-92. https://doi.org/10.1016/j.autcon.2008.05.004

Cong, H. P. N., Y. Choi. 2018. "Comparison of point cloud data and 3D CAD data for on-site dimensional inspection of industrial plant piping systems." Automation in Construction, 91, 44-52. https://doi.org/10.1016/j.autcon.2018.03.008

Demiralp, G., G. Guven, E. Ergen. 2012. “Analyzing the benefits of RFID technology for cost sharing in construction supply chains: A case study on prefabricated precast components." Automation in Construction, 24, 120-129. https://doi.org/10.1016/j.autcon.2012.02.005

Dey, T. K. 2007. Curve and surface reconstruction: algorithms with mathematical analysis, UK: Cambridge University Press.

Dimitrov A, Golparvar-Fard M. Segmentation of building point cloud models including detailed architectural/structural features and MEP systems[J]. Automation in Construction, 2015, 51: 32-45.

Edelsbrunner H, Kirkpatrick D, Seidel R. On the shape of a set of points in the plane [J]. IEEE Transactions on information theory, 1983, 29(4): 551-559.

Edis, E., I. Flores-Colen, J. De Brito. 2015. "Building Thermography: Detection of Delamination of Adhered Ceramic Claddings Using the Passive Approach." Journal of Nondestructive Evaluation, 34(1), 268. https://doi.org/10.1007/s10921-014-0268-2

Eulitz, M., G. Reiss. 2015. “3D reconstruction of SEM images by use of optical photogrammetry software.” Journal of Structural Biology, 191(2), 190-196. https://doi.org/10.1016/i.jsb.2015.06.010

Graham, K., L. Chow, S. Fai. 2018. "Level of detail, information and accuracy in building information modelling of existing and heritage buildings." Journal of Cultural Heritage Management and Sustainable Development, 8(4), 495-507. https://doi.org/10.1108/JCHMSD-09-2018-0067

Hackel, T., J. D. Wegner, K. Schindler. 2016. "Contour detection in unstructured 3d point clouds." In Proceedings of the IEEE Conference on Computer Vision and Pattern Recognition. 1610-1618. Las Vegas: IEEE. DOI: 


\section{$\underline{10.1109 / C V P R .2016 .178}$}

Jaillon, L., C. S. Poon, Y. H. Chiang. 2009. "Quantifying the waste reduction potential of using prefabrication in building construction in Hong Kong." Waste Management, 29 (1), 309-320. https://doi.org/10.1016/j.wasman.2008.02.015

Javidrad, F., A. R. Pourmoayed. 2011. "Contour curve reconstruction from cloud data for rapid prototyping." Robotics and Computer-Integrated Manufacturing. 27(2), 397-404. https://doi.org/10.1016/j.rcim.2010.08.008

Kalasapudi V S, Turkan Y, Tang P. Toward automated spatial change analysis of MEP components using 3D point clouds and as-designed BIM models[C]//2014 2nd International Conference on 3D Vision. IEEE, 2014, 2: 145152.

Kim M K, Wang Q, Park J W, et al. Automated dimensional quality assurance of full-scale precast concrete elements using laser scanning and BIM[J]. Automation in Construction, 2016, 72: 102-114.

Kovacs, L., A. Zimmermann, G. Brockmann, et al. 2006. "Accuracy and precision of the three-dimensional assessment of the facial surface using a 3-D laser scanner." IEEE transactions on medical imaging, 25(6), 742754. DOI: $10.1109 /$ TMI.2006.873624

Latimer, D., D. Gujar, J. Garrett, B. Akinci, S. Thayer, C. Paredis. 2002. ICES Research Project Report: Running surface assessment technology review. Carnegie Mellon University, Pittsburgh.

Li, C. Z., F. Xue, X. Li, J. L. Hong, G. Q. Shen. 2018. “An Internet of Things-enabled BIM platform for on-site assembly services in prefabricated construction." Automation in construction, 89, 146-161. https://doi.org/10.1016/j.autcon.2018.01.001

Li, C. Z., R. Y. Zhong, F. Xue, G. Xu, K. Chen, G. G. Huang, G. Q. Shen. 2017. "Integrating RFID and BIM technologies for mitigating risks and improving schedule performance of prefabricated house construction.” Journal of Cleaner Production, 165:1048-1062. https://doi.org/10.1016/j.jclepro.2017.07.156

Malihi, S., M. J. V. Zoej, M. Hahn, M. Mokhtarzade. 2018. "Window Detection from UAS-Derived Photogrammetric Point Cloud Employing Density-Based Filtering and Perceptual Organization.” Remote Sensing, 10(8),1320-1342. https://doi.org/10.3390/rs10081320

Nahangi, Mohammad, Thomas Czerniawski, Jamie Yeung, Carl T. Haas, Scott Walbridge and Jeffrey S. West. "An image-based frameworks for automated discrepancy quantification and realignment of industrial assemblies." (2015).

Park, H. S., H. M. Lee, A. Hojjat, I. Lee. 2007. “A new approach for health monitoring of structures: terrestrial laser scanning." Computer Aided Civil and Infrastructure Engineering. 22(1), 19-30. DOI: 10.1111/j.1467$\underline{8667.2006 .00466 . x}$ 
Pu, S., G. Vosselman. 2009. "Knowledge based reconstruction of building models from terrestrial laser scanning data." ISPRS Journal of Photogrammetry and Remote Sensing, 64(6), 575-584. https://doi.org/10.1016/j.isprsjprs.2009.04.001

Ramaji, I. J., A. M. Memari. 2015. "Information exchange standardization for BIM application to multi-story modular residential buildings." In Proceedings of the Architectural Engineering National Conference 2015 : Birth and Life of the Integrated Building, Milwaukee, WI, USA.

Rodríguez-Gonzálvez, P., M. Rodríguez-Martín, L. F. Ramos, D. González-Aguilera. “3D reconstruction methods and quality assessment for visual inspection of welds." Automation in Construction, 79, 49-58. https://doi.org/10.1016/j.autcon.2017.03.002

Sacks, R., A. Kedar, A. Borrmann, et al. 2018. "SeeBridge as next generation bridge inspection: overview, information delivery manual and model view definition." Automation in Construction, 90, 134-145. https://doi.org/10.1016/j.autcon.2018.02.033

Sambath, S., P. Nagaraj, N. Selvakumar, S. Arunachalam, T. Page. 2010. "Automatic detection of defects in ultrasonic testing using artificial neural network." International Journal of Microstructure and Materials Properties, 5(6), 561-574. DOI: 10.1504/IJMMP.2010.038155

Schnabel, R., R. Wahl, R. Klein. 2007. "Efficient RANSAC for point- cloud shape detection." In Computer graphics forum, 26(2), 214-226, Oxford, UK: Blackwell Publishing Ltd.

Shahzad, W. M., J. Mbachu, N. Domingo. 2014. "Prefab content versus cost and time savings in construction projects: A regression analysis." In 4th New Zealand Built Environment Research Symposium (NZBERS). Auckland, New Zealand.

Venugopal, M., C. M. Eastman, R. Sacks, J. Teizer. 2012. "Semantics of model views for information exchanges using the industry foundation class schema." Advanced Engineering Informatics, 26 (2), 411-428.

Wack R, Wimmer A. Digital terrain models from airborne laser scanner data-a grid based approach[J]. International Archives of Photogrammetry Remote Sensing and Spatial Information Sciences, 2002, 34(3/B): 293-296.

Wang C, Cho Y K, Kim C. Automatic BIM component extraction from point clouds of existing buildings for sustainability applications[J]. Automation in Construction, 2015, 56: 1-13.

Wang Q, Guo J, Kim M K. An application oriented scan-to-BIM framework[J]. Remote sensing, 2019, $11(3)$ : 365.

Zhou Z, Gong J, Guo M. Image-based 3D reconstruction for posthurricane residential building damage assessment[J]. Journal of Computing in Civil Engineering, 2016, 30(2): 04015015. 
Table 1: Allowable deviation of the dimensions of precast concrete component

\begin{tabular}{|c|c|c|c|c|c|}
\hline \multirow{3}{*}{$\begin{array}{c}\text { Prefabricated } \\
\text { column }\end{array}$} & Length & \pm 3 & \pm 5 & \pm 7 & \pm 10 \\
\hline & Width & \pm 3 & \pm 5 & \pm 7 & \pm 10 \\
\hline & Height & \pm 2 & \pm 3 & \pm 4 & \pm 6 \\
\hline \multirow{3}{*}{ Prefabricated beam } & Length & \pm 3 & \pm 5 & \pm 7 & \pm 10 \\
\hline & Width & \pm 3 & \pm 5 & \pm 7 & \pm 10 \\
\hline & Thickness & \pm 2 & \pm 3 & \pm 4 & \pm 6 \\
\hline \multirow{4}{*}{ Prefabricated slab } & Length & \pm 3 & \pm 5 & \pm 7 & \pm 10 \\
\hline & Width & \pm 3 & \pm 5 & \pm 7 & \pm 10 \\
\hline & Thickness & \pm 2 & \pm 3 & \pm 4 & \pm 6 \\
\hline & Diagonal length & \pm 6 & \pm 8 & \pm 10 & \pm 12 \\
\hline \multirow{4}{*}{$\begin{array}{c}\text { Prefabricated } \\
\text { interior wall }\end{array}$} & Length & \pm 3 & \pm 4 & \pm 5 & \pm 6 \\
\hline & Width & \pm 3 & \pm 4 & \pm 5 & \pm 6 \\
\hline & Thickness & \pm 2 & \pm 3 & \pm 4 & \pm 5 \\
\hline & Diagonal length & \pm 3 & \pm 4 & \pm 5 & \pm 5 \\
\hline \multirow{4}{*}{$\begin{array}{l}\text { Prefabricated } \\
\text { external wall }\end{array}$} & Length & \pm 3 & \pm 3 & \pm 4 & \pm 4 \\
\hline & Width & \pm 3 & \pm 3 & \pm 4 & \pm 4 \\
\hline & Thickness & \pm 2 & \pm 2 & \pm 3 & \pm 3 \\
\hline & Diagonal length & \pm 3 & \pm 4 & \pm 5 & \pm 5 \\
\hline \multirow{3}{*}{ Prefabricated stair } & Length & \pm 3 & - & - & - \\
\hline & Width & \pm 3 & - & - & - \\
\hline & Thickness & \pm 2 & - & - & - \\
\hline \multirow{2}{*}{ Reserved hole } & Offset of center line & 5 & - & - & - \\
\hline & Size & \pm 5 & - & - & - \\
\hline
\end{tabular}

Table 2: Allowable surface defect for prefabricated concrete components

\begin{tabular}{|c|c|}
\hline Defect type & Allowance \\
\hline \multirow[t]{2}{*}{ Crack } & Width $<0.02 \mathrm{~mm} \&$ Length $<100 \mathrm{~mm}$ \\
\hline & The area in the secondary part $<10000 \mathrm{~mm}^{2}$ or the proportion of the \\
\hline \multicolumn{2}{|l|}{ Honeycomb } \\
\hline & area $<0.5 \%$ \\
\hline Collapse of edge & The proportion of the plane area $<0.5 \%$ or the number $<3$ \\
\hline Surface voids & The proportion of the plane area $<1 \%$ \\
\hline Reinforcement exposure & Should not exist \\
\hline Defect of the connection & Should not exist \\
\hline
\end{tabular}


Table 3: Comparison of different $K$ values

\begin{tabular}{ccccccc}
\hline The K value & $\begin{array}{c}\text { 1(The original } \\
\text { data) }\end{array}$ & $\mathbf{2}$ & $\mathbf{3}$ & $\mathbf{4}$ & $\mathbf{5}$ & $\mathbf{6}$ \\
\hline The number of points & 582,357 & 380,565 & 362,596 & 350,591 & 368,967 & 380,848 \\
Compression ratio & $100 \%$ & $65.35 \%$ & $62.26 \%$ & $60.20 \%$ & $63.36 \%$ & $65.40 \%$ \\
\hline
\end{tabular}

Table 4: Weights of production quality evaluation index for precast concrete

\begin{tabular}{cccccc}
\hline First-class index & $\begin{array}{c}\text { Assign } \\
\text { weight }\end{array}$ & Second-class index & $\begin{array}{c}\text { Assign } \\
\text { weight }\end{array}$ & $\begin{array}{c}\text { Comprehensive } \\
\text { weight }\end{array}$ & Value \\
\hline \multirow{3}{*}{$\begin{array}{c}\text { Production } \\
\text { accuracy }\end{array}$} & \multirow{2}{*}{0.6} & Length accuracy & 0.4 & 0.24 & $0 / 1$ \\
& & Angle accuracy & 0.3 & 0.18 & $0 / 1$ \\
& & Positioning accuracy of reserved hole & 0.2 & 0.12 & $0 / 1$ \\
& & Shape accuracy of reserved hole & 0.1 & 0.06 & $0 / 1$ \\
Surface quality & Crack & 0.3 & 0.12 & $0 / 1$ \\
& \multirow{2}{*}{0.4} & Honeycomb & 0.1 & 0.04 & $0 / 1$ \\
& & Angular collapse & 0.1 & 0.04 & $0 / 1$ \\
& & Surface pockmark & 0.1 & 0.04 & $0 / 1$ \\
& & Leakage reinforcement & 0.2 & 0.08 & $0 / 1$ \\
& & Joint defect & 0.2 & 0.08 & $0 / 1$ \\
\hline
\end{tabular}


Table 5: Calculation example of comprehensive evaluation index

\begin{tabular}{|c|c|c|c|c|c|c|}
\hline \multirow[t]{2}{*}{$\begin{array}{l}\text { Type of } \\
\text { element }\end{array}$} & \multirow[t]{2}{*}{ Specific index } & \multicolumn{2}{|c|}{ Requirements of index } & \multirow{2}{*}{$\begin{array}{c}\text { Actual data } \\
2.22\end{array}$} & \multirow[t]{2}{*}{$\begin{array}{c}\text { Index } \\
\text { weight }\end{array}$} & \multirow{2}{*}{$\begin{array}{c}\begin{array}{c}\text { Index } \\
\text { value }\end{array} \\
1\end{array}$} \\
\hline & & Length & \pm 3 & & & \\
\hline \multirow{11}{*}{ 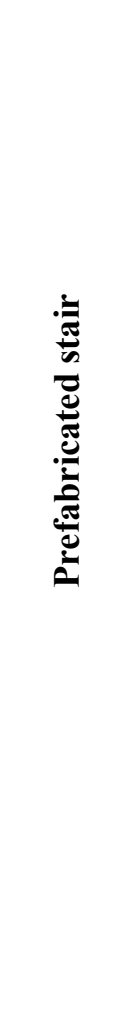 } & Length accuracy & Width & \pm 3 & -0.17 & 0.24 & 1 \\
\hline & & Thickness & \pm 2 & -2.84 & & 1 \\
\hline & Angle accuracy & & & - & 0.18 & 1 \\
\hline & $\begin{array}{l}\text { Positioning accuracy of } \\
\text { reserved hole }\end{array}$ & $\begin{array}{l}\text { Offset of center } \\
\text { line position }\end{array}$ & 5 & 2.15 & 0.12 & 1 \\
\hline & $\begin{array}{l}\text { Shape accuracy of reserved } \\
\text { hole }\end{array}$ & Size & \pm 5 & 0.55 & 0.06 & 1 \\
\hline & Crack & \multicolumn{2}{|c|}{$\begin{array}{l}\text { Width is less than } 0.02 \mathrm{~mm} \text { and length } \\
\text { is less than } 100 \mathrm{~mm}\end{array}$} & Not detected & 0.12 & 1 \\
\hline & Honeycomb & $\begin{array}{l}\text { The secondar } \\
10000 \mathrm{~mm}^{2} \text { or } \mathrm{t}\end{array}$ & $\begin{array}{l}\text { ess than } \\
\text { less than }\end{array}$ & Not detected & 0.04 & 1 \\
\hline & Angular collapse & \multicolumn{2}{|c|}{$\begin{array}{l}\text { Plane area ratio is less than } 0.5 \% \text { / less } \\
\text { than } 3\end{array}$} & $0.446 \% / 3$ & 0.04 & 0 \\
\hline & Surface pockmark & \multicolumn{2}{|c|}{ Plane area ratio is less than $1 \%$} & Not detected & 0.04 & 1 \\
\hline & Leakage reinforcement & \multicolumn{2}{|c|}{ There should be no } & Not detected & 0.08 & 1 \\
\hline & Joint defect & \multicolumn{2}{|c|}{ There should be no } & Not detected & 0.08 & 1 \\
\hline
\end{tabular}

Table 6: Concrete prefabricated wall property set definition

\begin{tabular}{cc}
\hline PsetName & PSet_PrefabricateWall \\
Entity & IfcWall \\
PropertyType & IfcWall/Userdefined/PrefabricateWall \\
PropertyDescribe & Used to describe the quality of concrete prefabricated walls \\
\hline
\end{tabular}


Table 7: Property sets for quality of concrete prefabricated components

\begin{tabular}{cccc}
\hline & PropertyName & PropertyValue & Type \\
\hline $\mathbf{1}$ & Length Deviation & IfcPropertySingleValue & IfcReal \\
$\mathbf{2}$ & Width Deviation & IfcPropertySingleValue & IfcReal \\
$\mathbf{3}$ & Height Thickness Deviation & IfcPropertySingleValue & IfcReal \\
$\mathbf{4}$ & Diagonal Deviation & IfcPropertySingleValue & IfcReal \\
$\mathbf{5}$ & Reserved Hole Position & IfcPropertySingleValue & IfcReal \\
$\mathbf{6}$ & Deviation & IfcPropertySingleValue & IfcReal \\
$\mathbf{7}$ & Crack & IfcPropertySingleValue & IfcReal \\
$\mathbf{8}$ & Honeycomb & IfcPropertySingleValue & IfcReal \\
$\mathbf{9}$ & Corner Breakage & IfcPropertySingleValue & IfcReal \\
$\mathbf{1 0}$ & Pockmark & IfcPropertySingleValue & IfcReal \\
& & & IfcString \\
$\mathbf{1 1}$ & Reinforcing Bar Exposed & IfcPropertyEnumeratedValue & ( Y/N ) \\
& & & IfcString \\
& & & ( Y/N ) \\
$\mathbf{1 2}$ & Connection Reliability & IfcPropertyEnumeratedValue & IfcReal \\
\hline
\end{tabular}



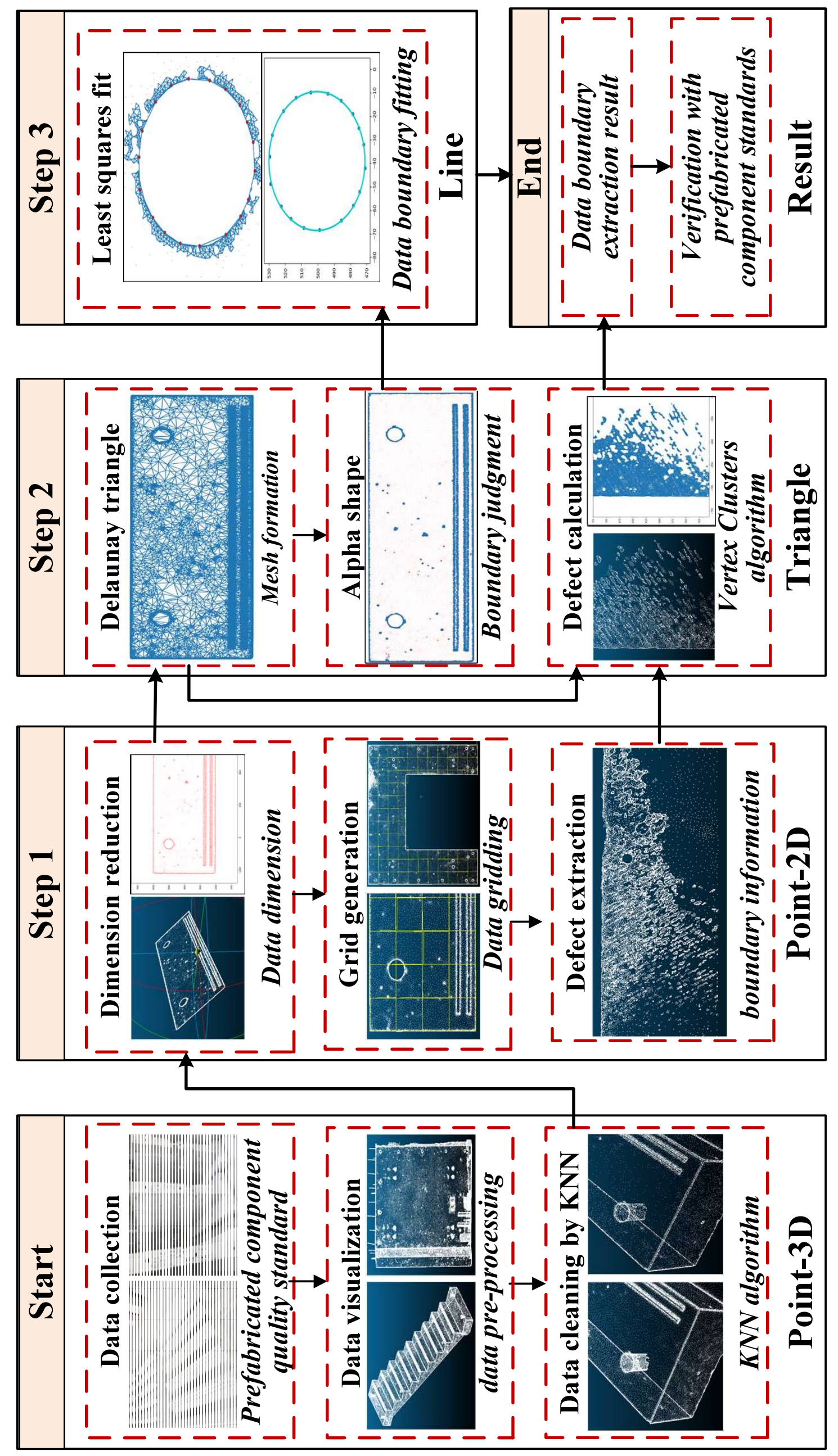


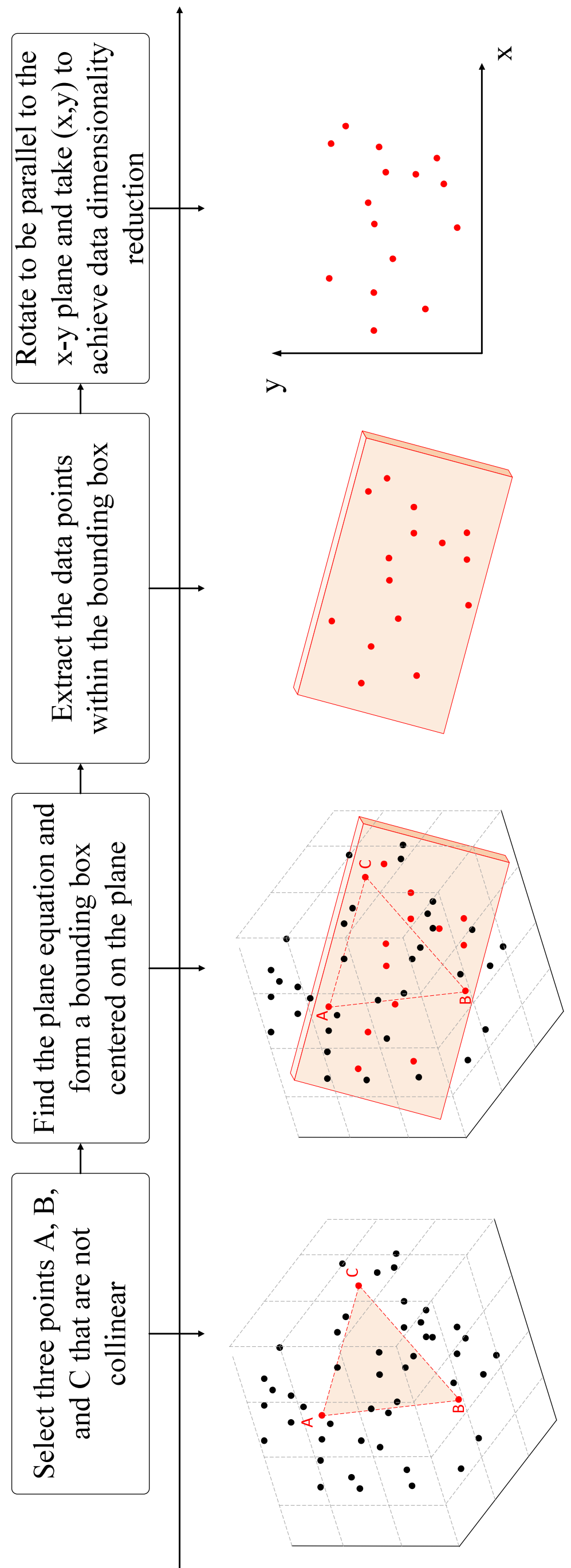




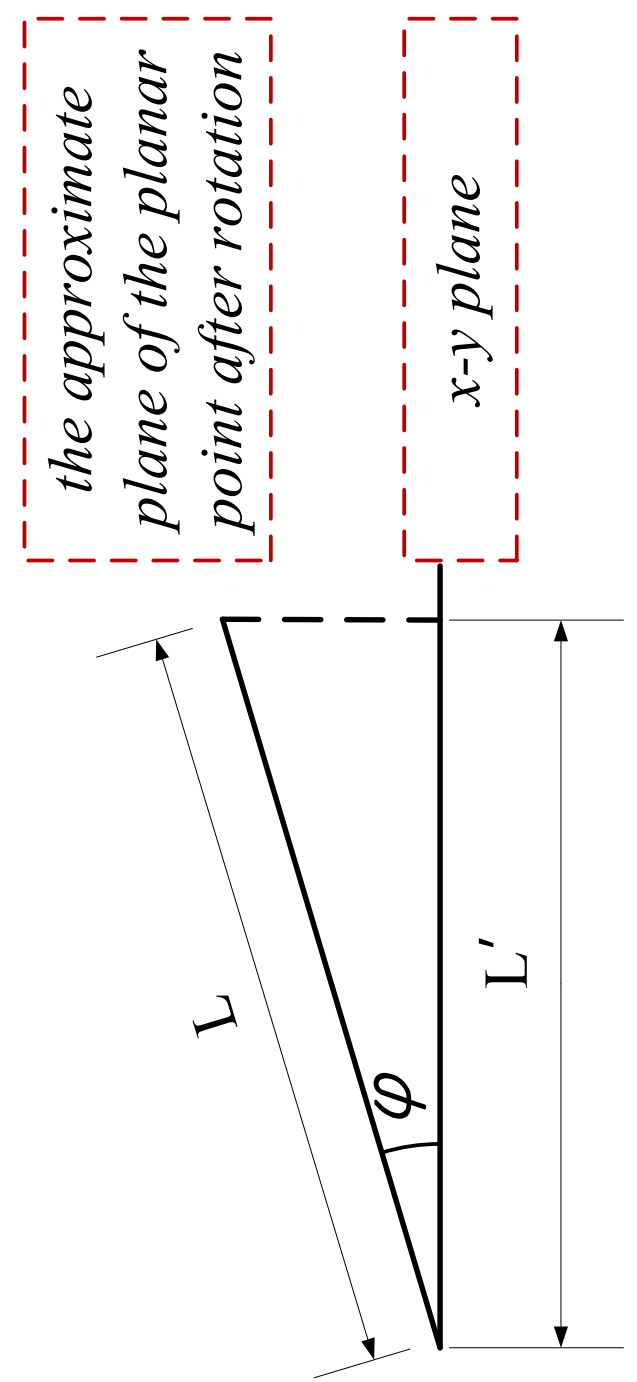



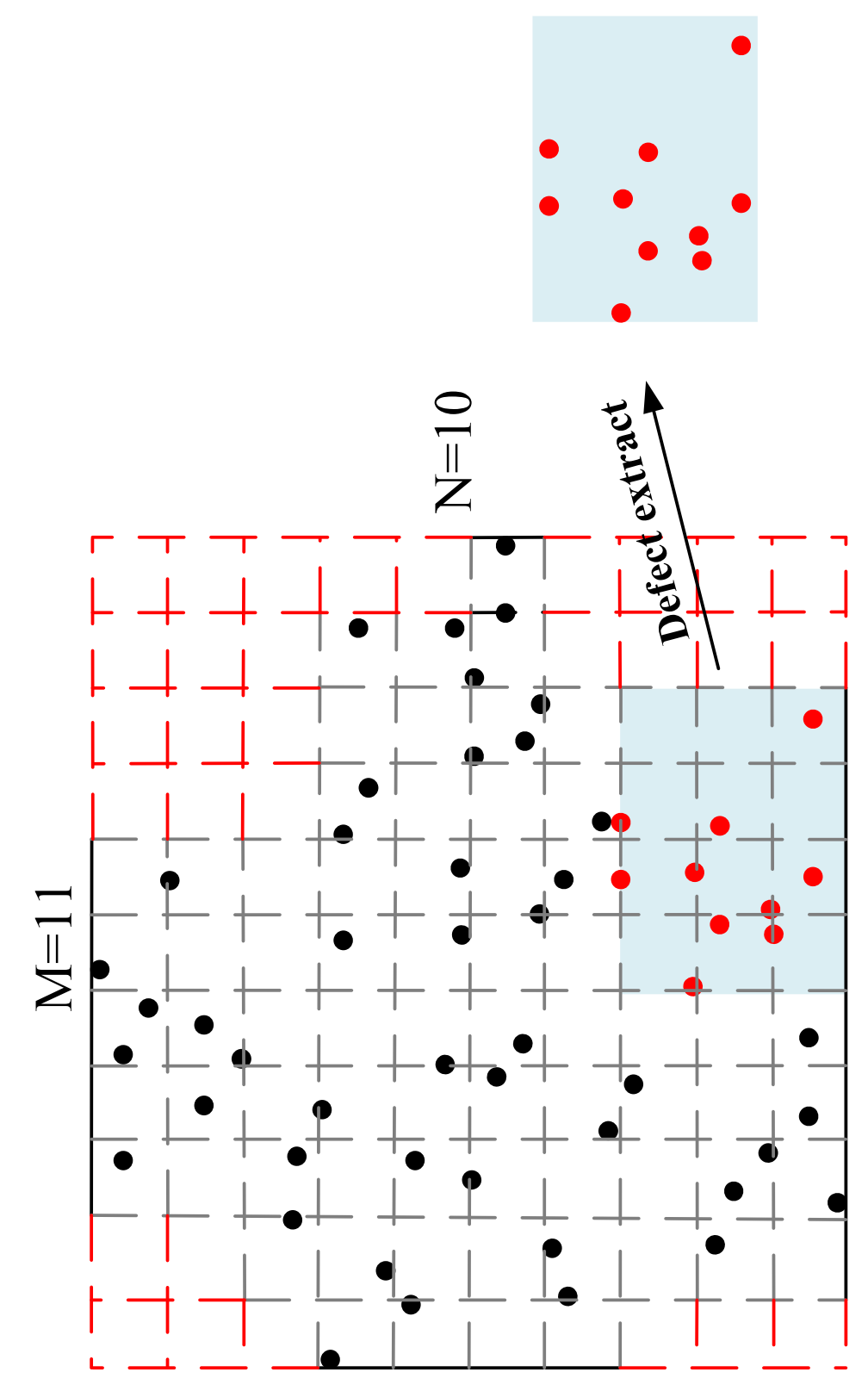


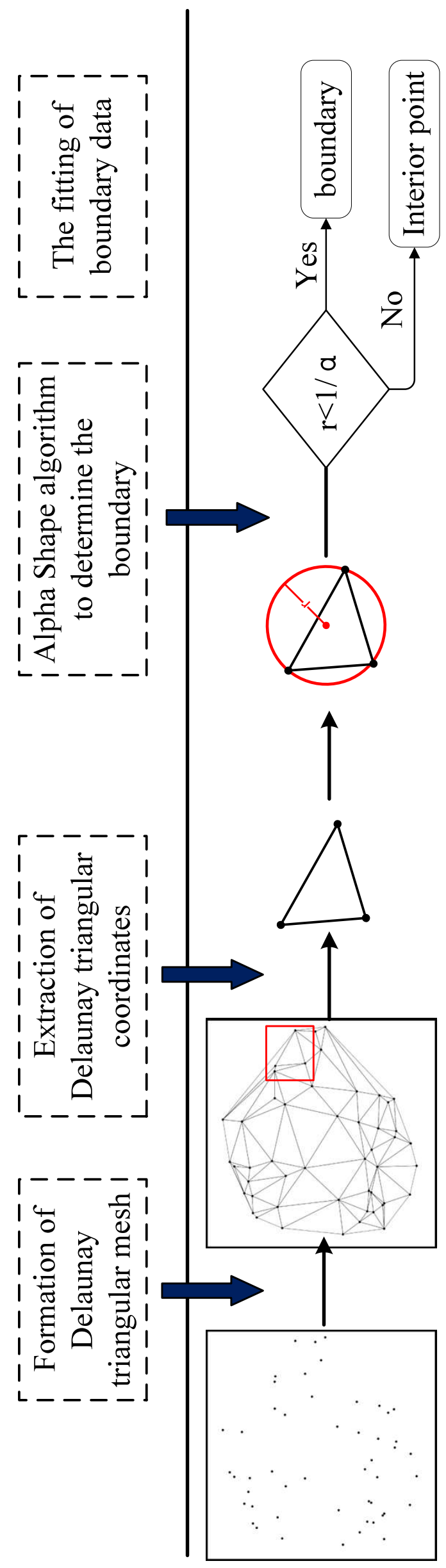

م
$\mathbb{0}$
$\frac{1}{5}$
믄 

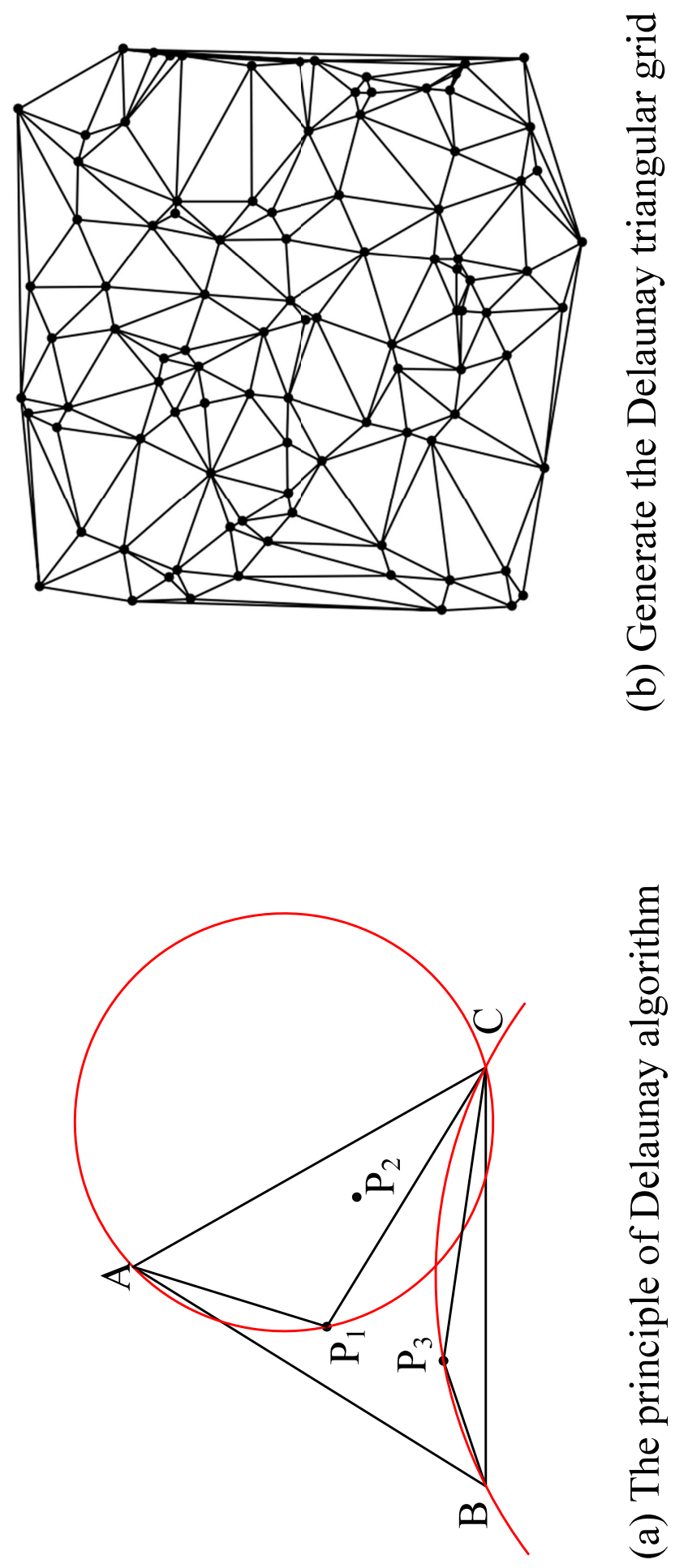

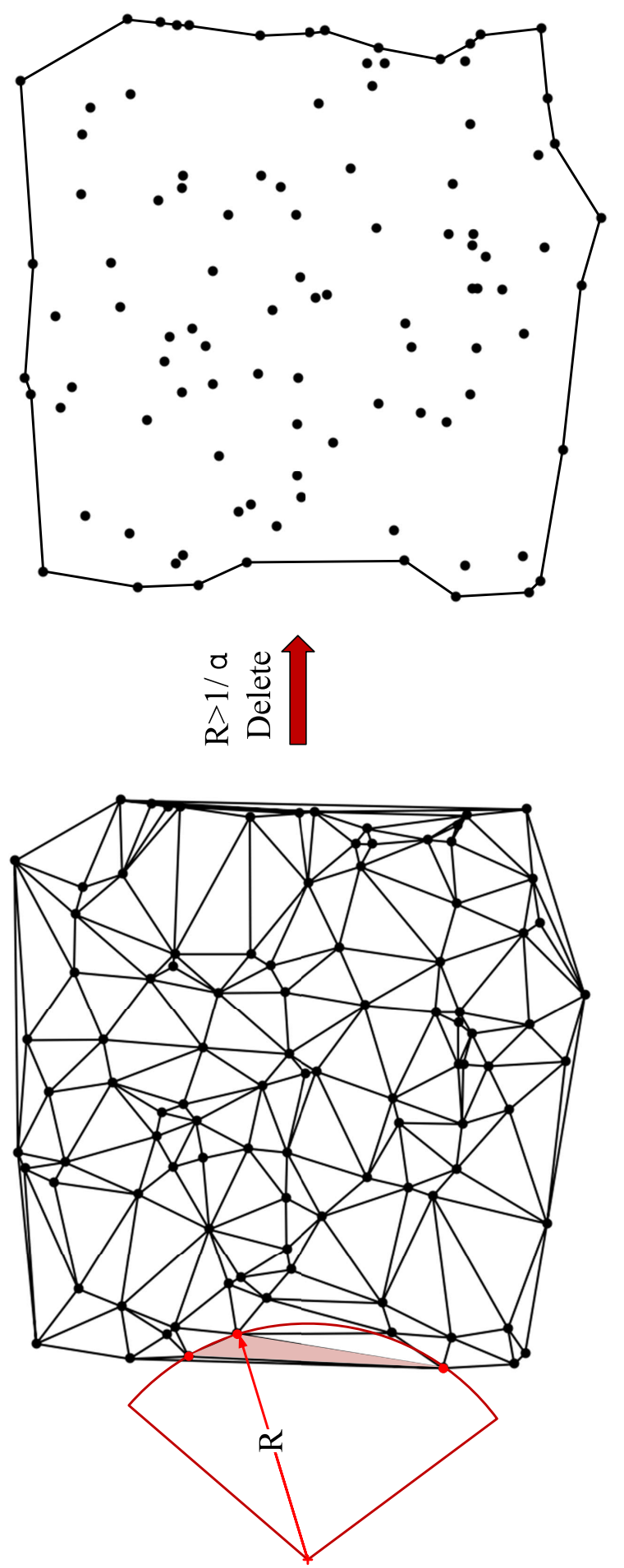


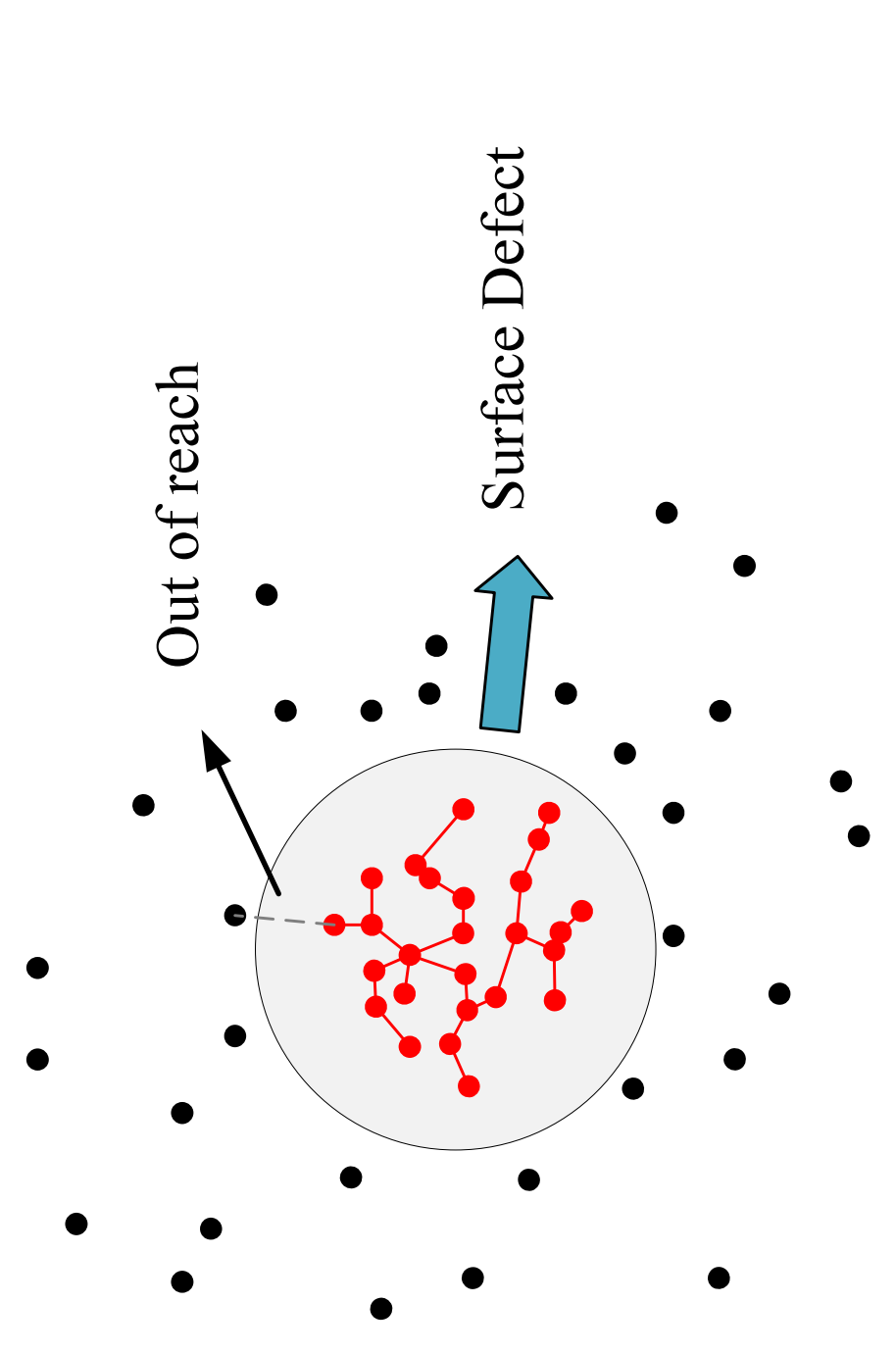

$\infty$
$\frac{0}{\square}$
$\frac{0}{2}$
$\frac{0}{4}$ 


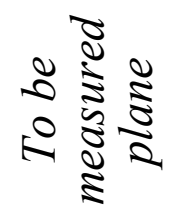

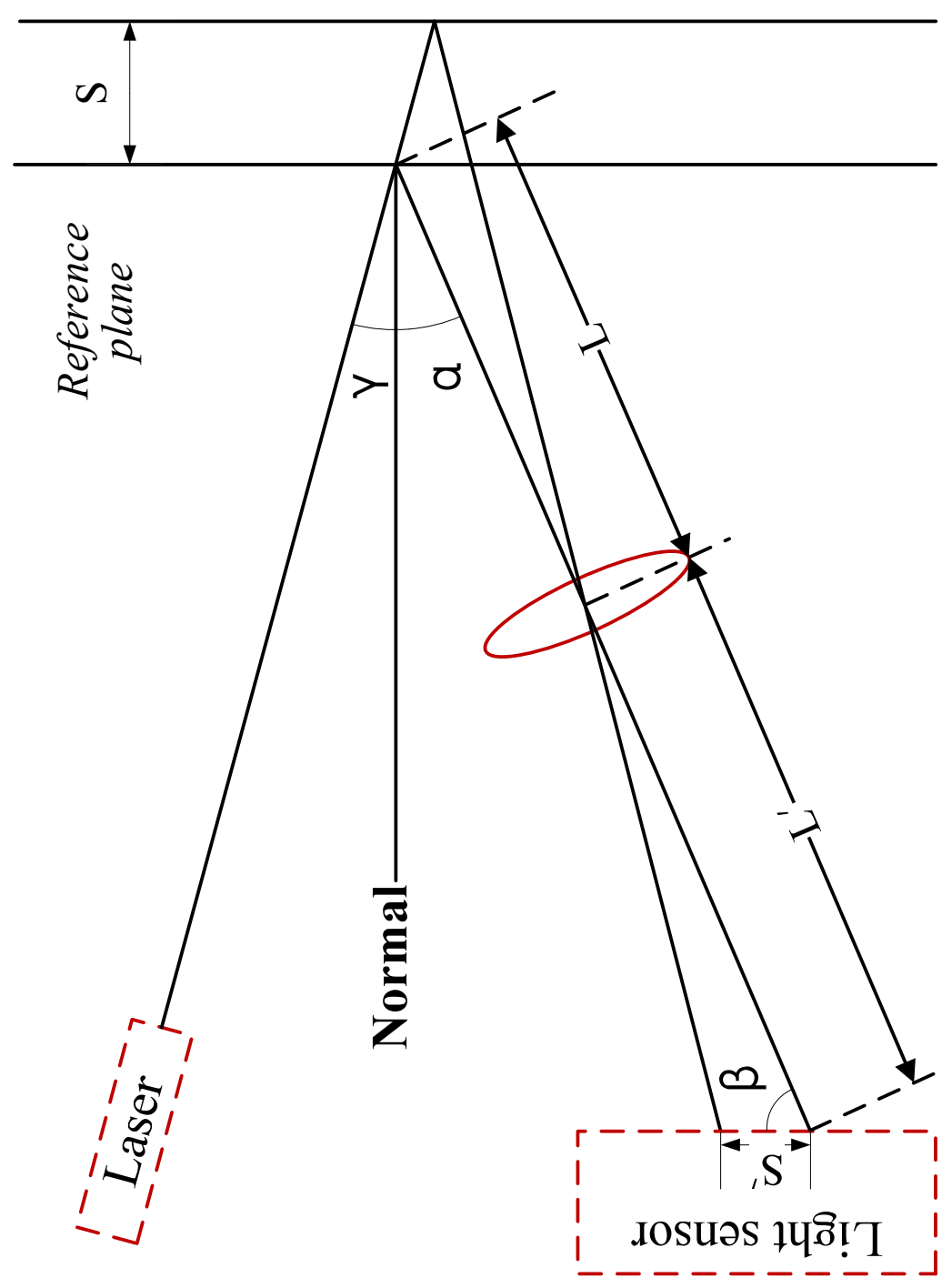



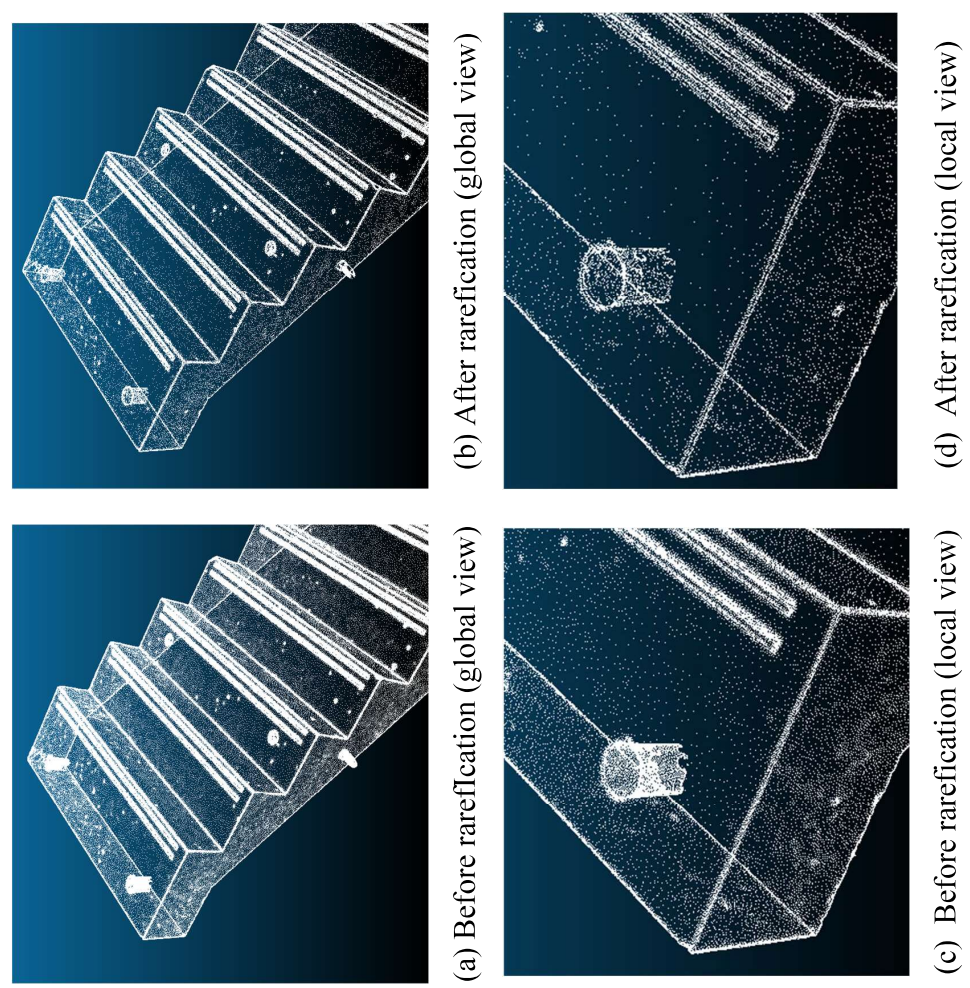

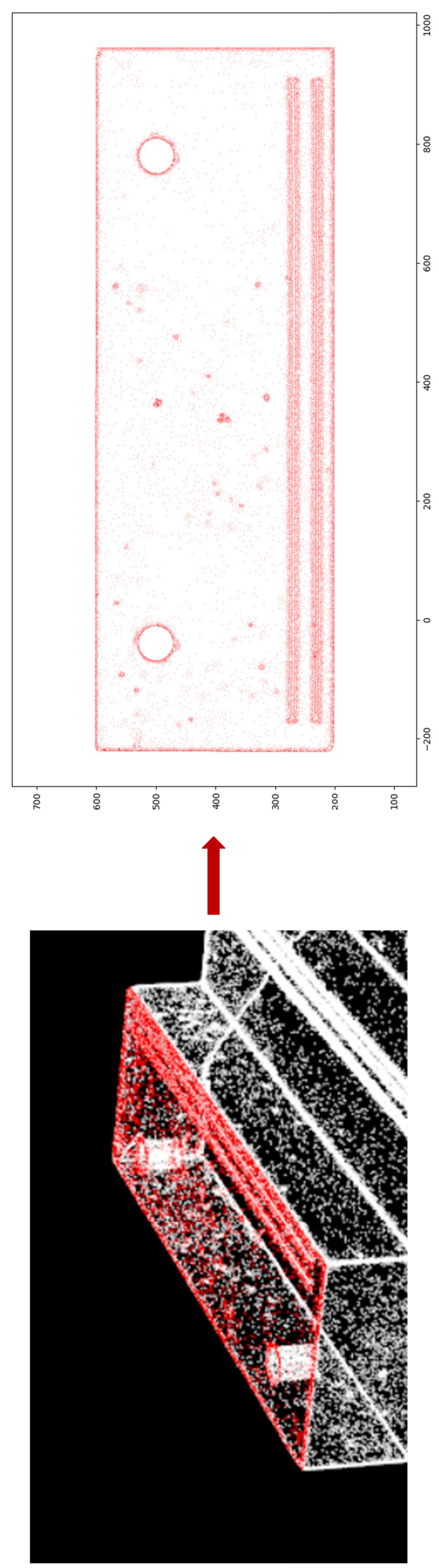

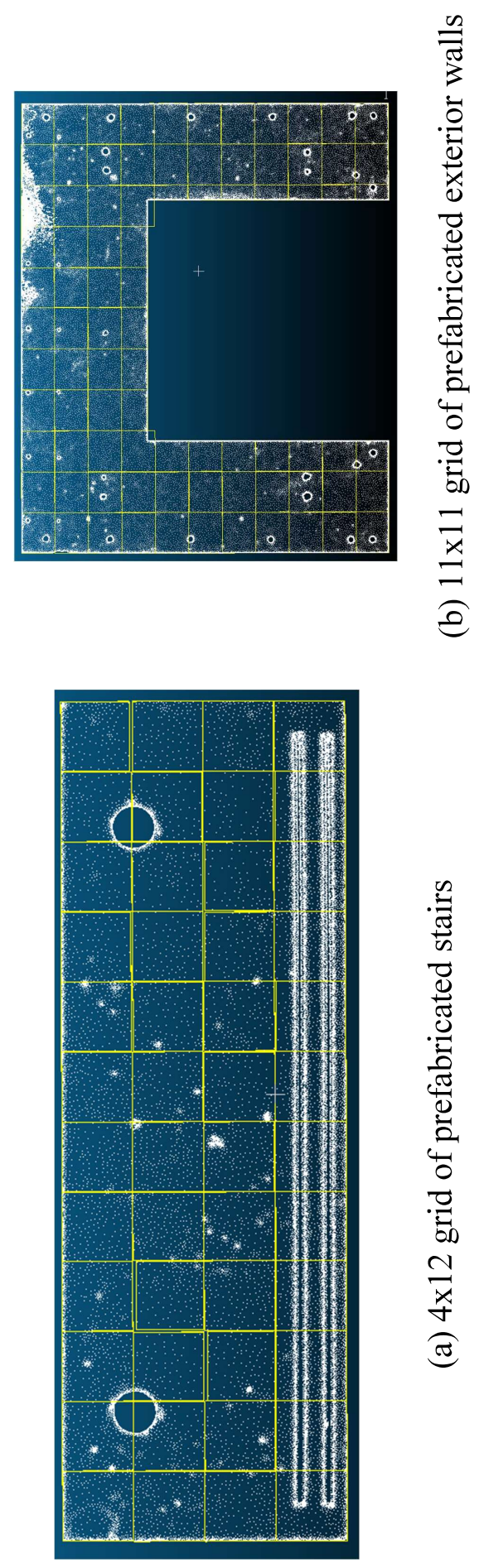

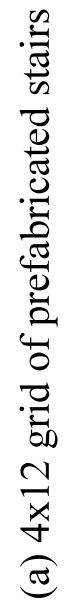



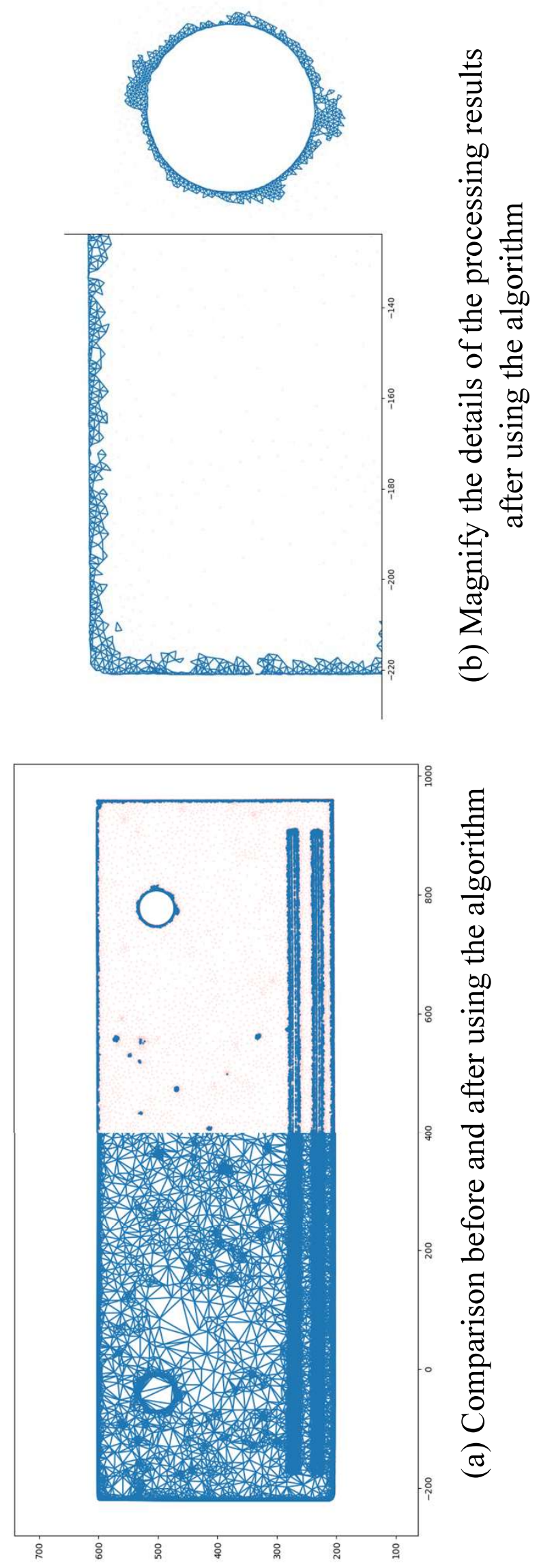


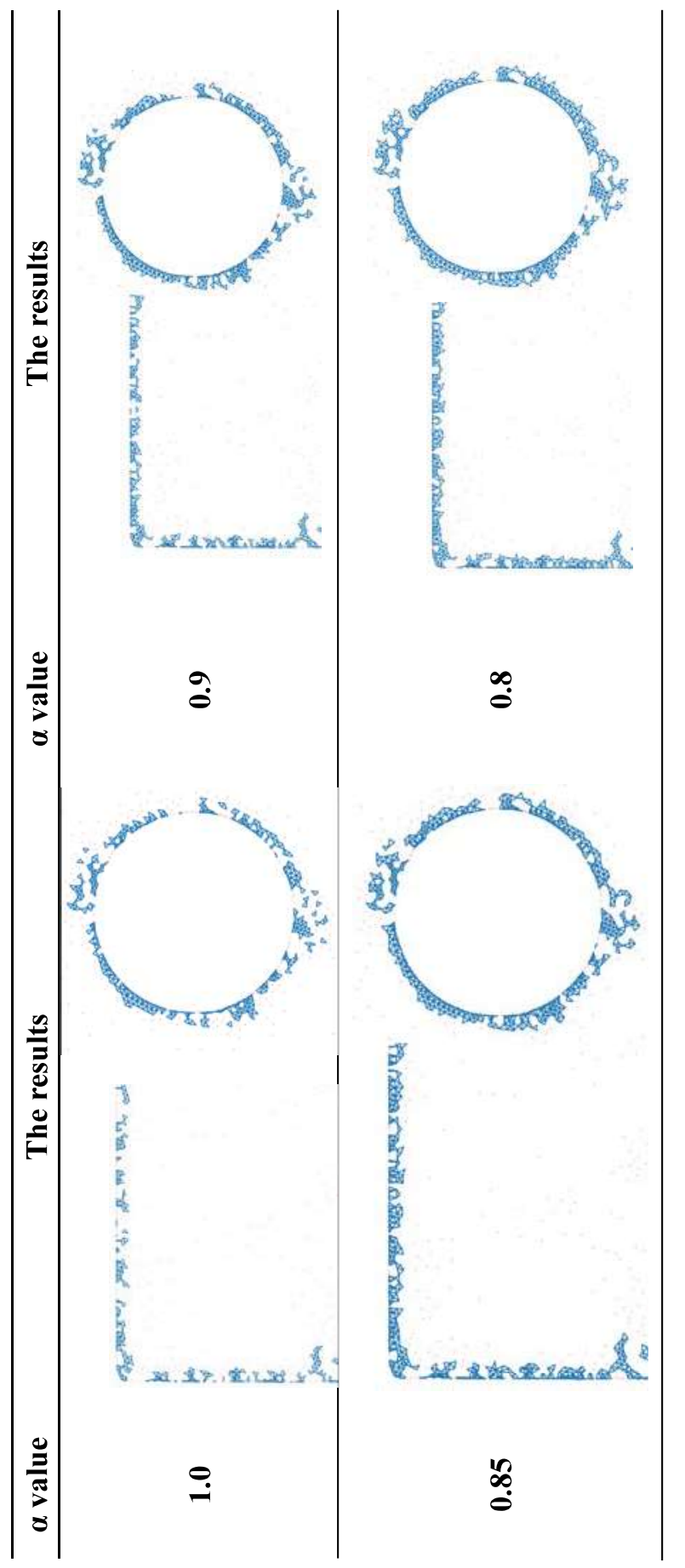




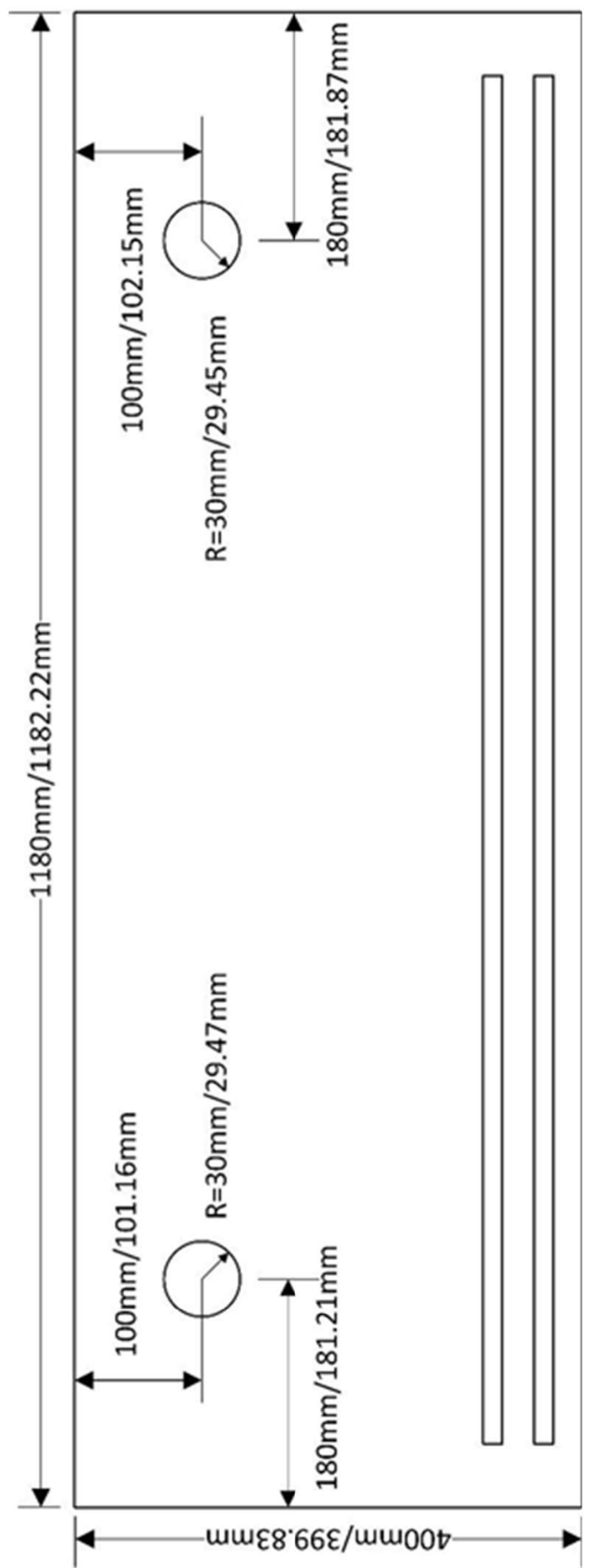

$\stackrel{10}{0}$
$\frac{0}{5}$
$\frac{\sqrt{6}}{4}$ 

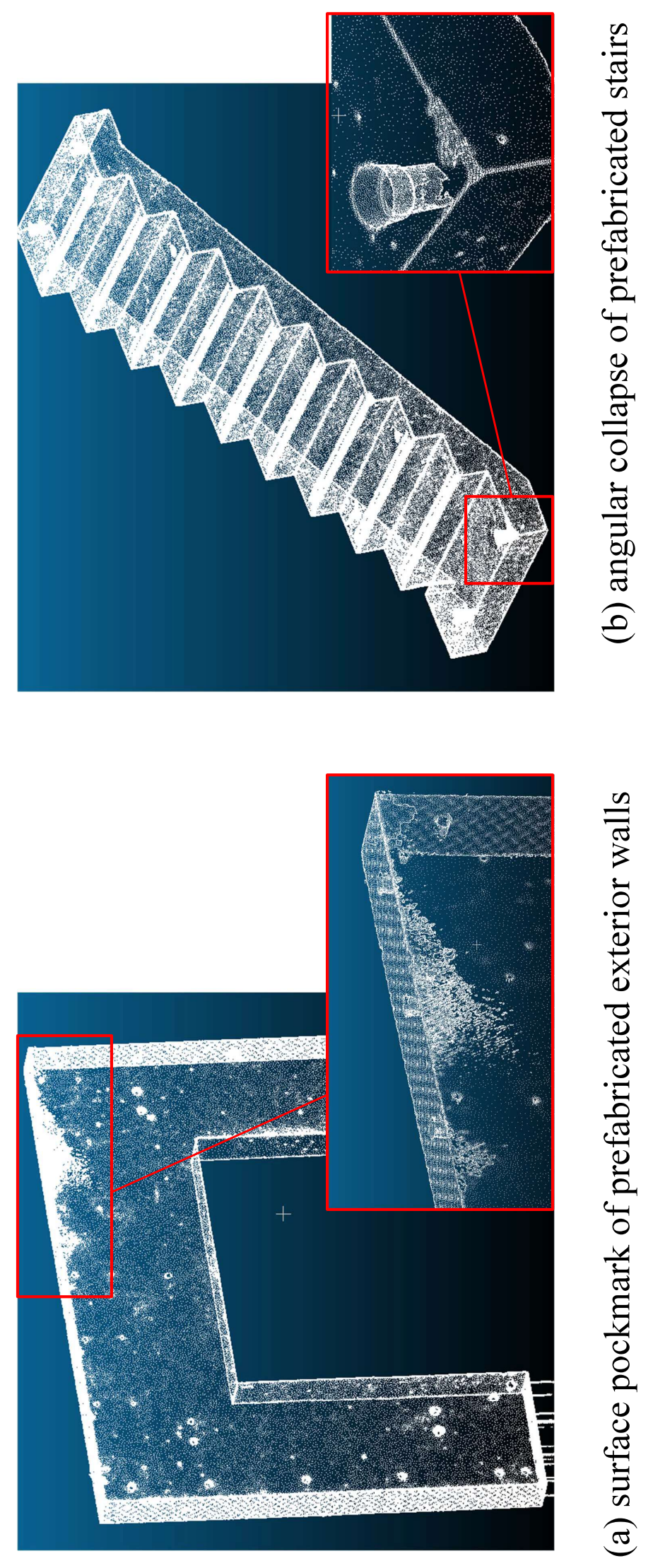

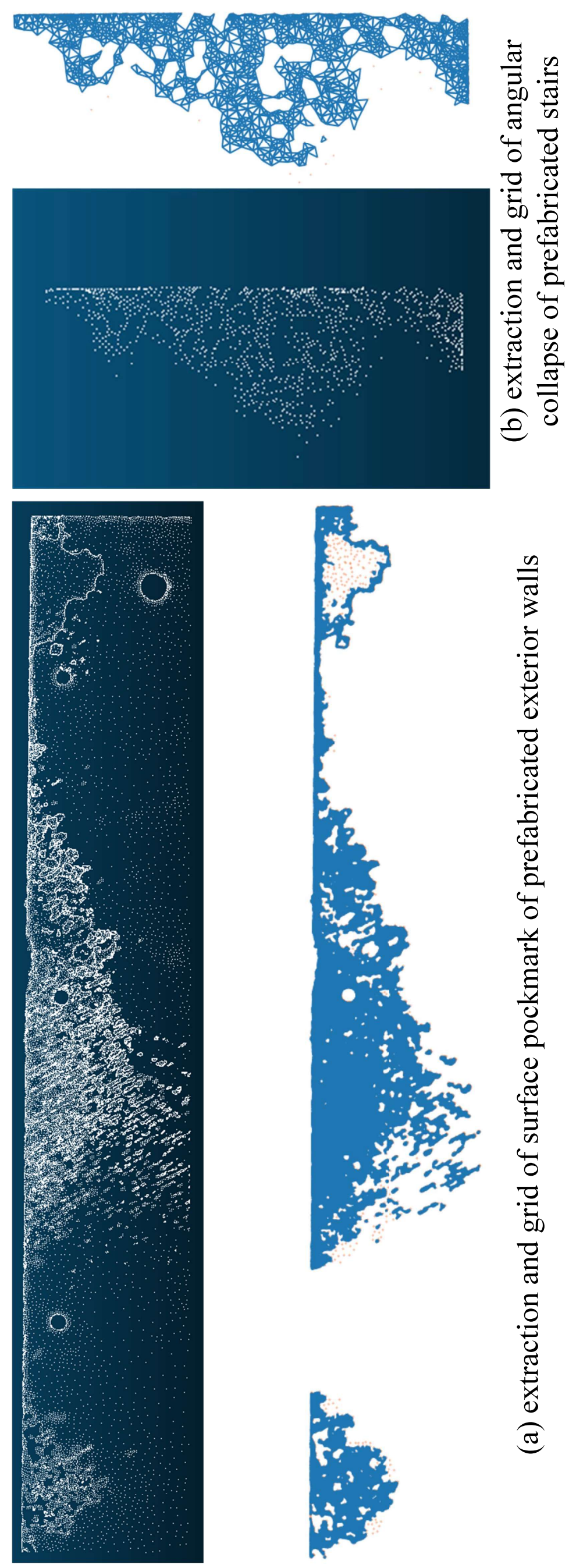

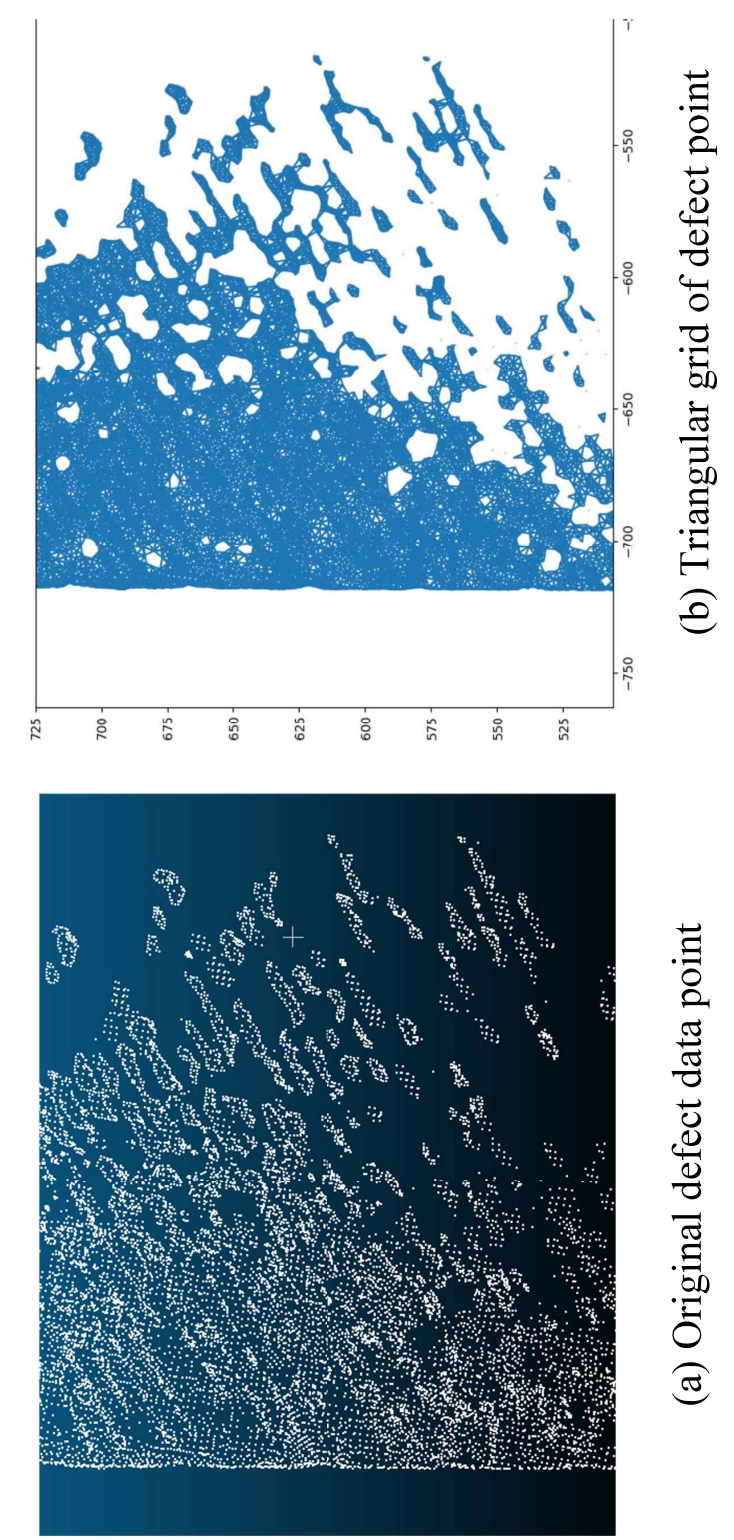


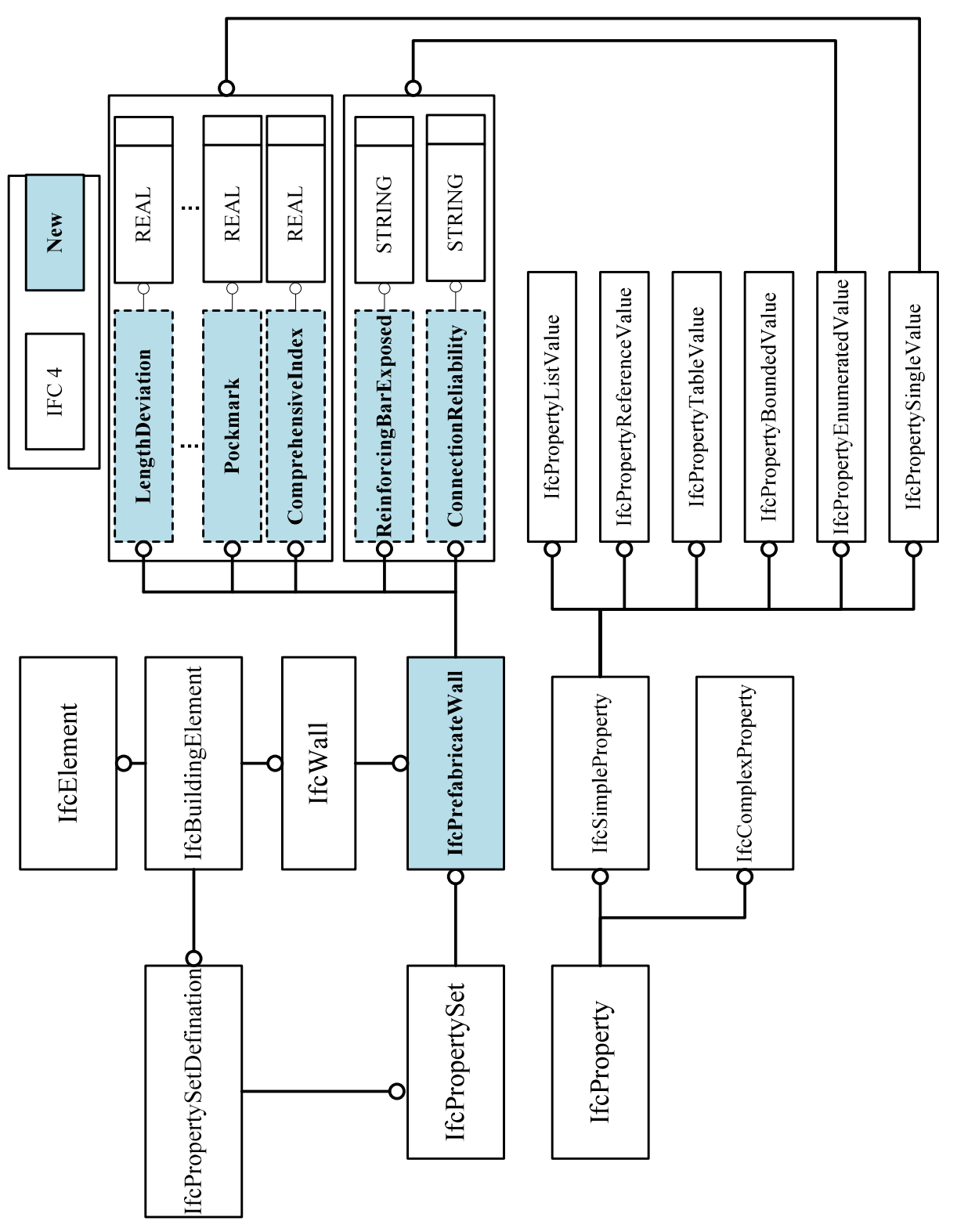




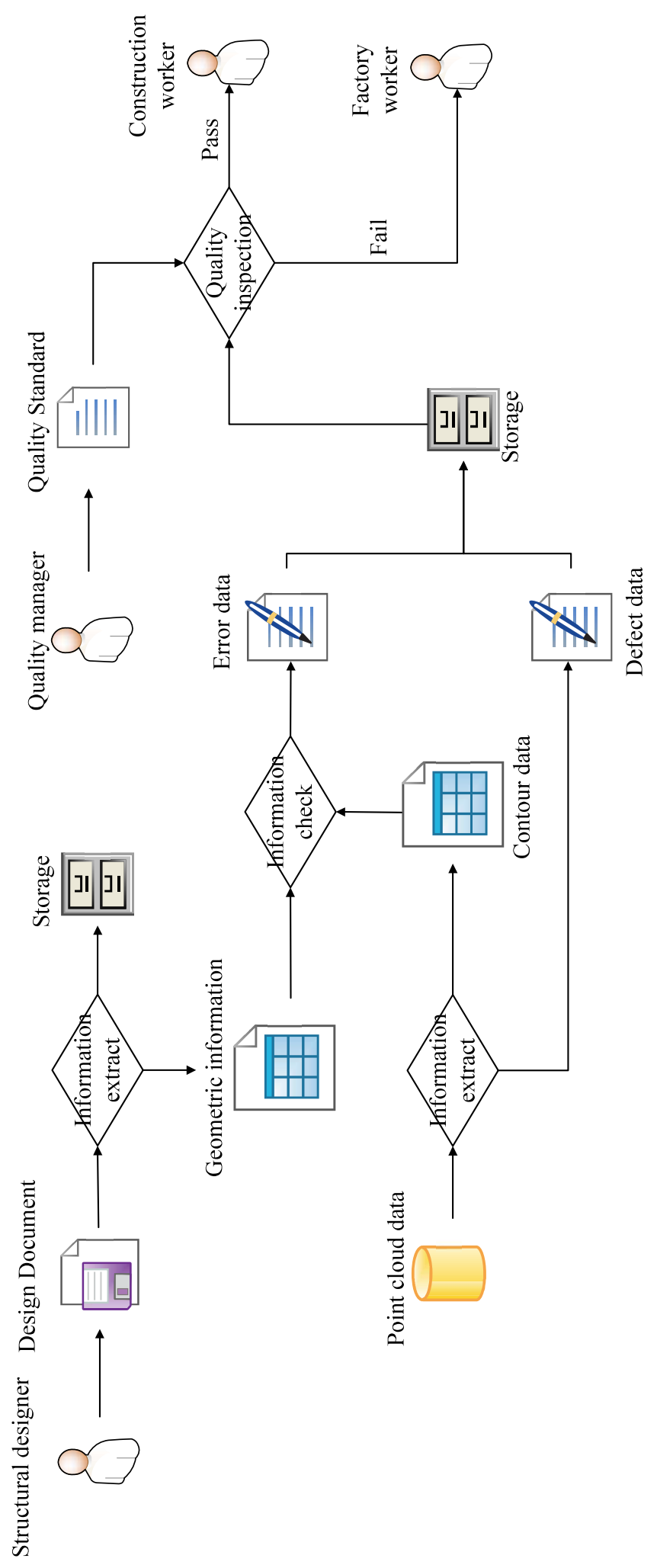




\section{Figure Caption List}

Figure 1: Overall research methodology

Figure 2: The technical route of data dimension reduction

Figure 3: Rotation error

Figure 4: Gridding of precast concrete components

Figure 5: Processing flow of boundary information

Figure 6: The principle and results of Delaunay grid generation

Figure 6a: The principle of Delaunay grid generation

Figure 6b: Generate Delaunay triangular grid

Figure 7: The results of using Alpha Shape algorithm

Figure 8: Separating defect points using Vertex Clusters algorithm

Figure 9: laser triangulation diagram

Figure 10: Comparison of the effect of down sampling

Figure 10a: Before rarefication (global view)

Figure 10b: After rarefication (global view)

Figure 10a: Before rarefication (local view)

Figure 10b: After rarefication (local view)

Figure 11: Dimension reduction result

Figure 12: Results of data gridding

Figure 12a: $4 \times 12$ grid of prefabricated stairs

Figure 12a: $11 \times 11$ grid of prefabricated exterior walls

Figure 13: The results of using Alpha Shape algorithm 
Figure 13a: Comparison before and after using the algorithm

Figure 13b: Magnify the details of the processing results after using the algorithm

Figure 14: Comparison of different $\alpha$ values

Figure 15: Step size error information of top surface of prefabricated stairs

Figure 16: Surface defect of prefabricated element

Figure 16a: Surface pockmark of prefabricated exterior walls

Figure 16b: Angular collapse of prefabricated stairs

Figure 17: Extraction and grid of defect area

Figure 17a: extraction and grid of surface pockmark of prefabricated exterior walls

Figure 17b: extraction and grid of angular collapse of prefabricated stairs

Figure 18: Defect point connection result

Figure 18a: Original defect data points

Figure 18b: Triangular grid of defect points

Figure 19: IFC extension of prefabricated wall using EXPRESS-G

Figure 20: The flowchart of the application process with point cloud data 